\title{
How the Affective Quality of Social Connections May Contribute to Public Health: Prosocial Tendencies Account for the Links Between Positivity Resonance and Behaviors that Reduce the Spread of COVID-19
}

\author{
Taylor N. West ${ }^{1}$ (D) $\cdot$ Khoa Le Nguyen $^{1} \cdot$ Jieni Zhou ${ }^{1} \cdot$ Michael M. Prinzing $^{2} \cdot$ Jenna L. Wells $^{3} \cdot$ Barbara L. Fredrickson $^{1}$ (D)
}

Received: 1 August 2020 / Accepted: 2 February 2021 / Published online: 13 April 2021

(C) The Society for Affective Science 2021

\begin{abstract}
Although behaviors such as handwashing, mask wearing, and social distancing are known to limit viral spread, early in the COVID-19 pandemic, many individuals in the United States did not adopt them. The positivity resonance theory of coexperienced positive affect (Fredrickson, 2016) holds that shared pleasant states that include the key features of mutual care and a sense of oneness through behavioral synchrony function to build prosocial tendencies (e.g., self-transcendent and otheroriented dispositions of felt unity, empathy, altruism, and general positivity toward humanity). We tested the theory-driven hypothesis that prosocial tendencies are associated with high-quality social connections characterized by the affective state of positivity resonance and, in turn, account for behaviors to slow the spread of COVID-19. We measured perceived positivity resonance at the level of social episodes either during the COVID-19 pandemic (study 1, $N=1059$, April-May 2020) or before it (study 2, $N=227$, March-November 2019). In both studies, cross-sectionally and prospectively, results suggest that perceived positivity resonance had a positive indirect effect on self-reported hygienic behaviors (e.g., handwashing and mask wearing), which was mediated by a latent measure of prosocial tendencies. Sensitivity analyses confirmed these mediation effects to be independent of competing predictors of prosocial tendencies (e.g., overall positive and negative affect, frequency of social interaction) and competing predictors of health behaviors (e.g., political orientation, high-risk status, illness symptoms). Effects for social distancing were mixed. Overall, findings are consistent with the view that positivity resonance builds selftranscendent prosocial tendencies that motivate behaviors to protect community health.
\end{abstract}

Keywords Positive psychology $\cdot$ Broaden-and-build theory $\cdot$ Health behavior $\cdot$ Emotion

In step with the rise of large-scale civilizations, humans have endured pandemics, a reality made salient in early 2020 as COVID-19, the disease caused by SARS-CoV-2, reached

Taylor N. West and Khoa Le Nguyen shared first authorship

Handling Editor: David Almeida

Barbara L. Fredrickson

blf@unc.edu

1 Department of Psychology and Neuroscience, University of North Carolina at Chapel Hill, 309 Davie Hall, Campus Box 3270, Chapel Hill, NC 27599-3720, USA

2 Department of Philosophy, University of North Carolina at Chapel Hill, Chapel Hill, NC, USA

3 Department of Psychology, University of California, Berkeley, Berkeley, CA, USA multiple continents. Absent vaccines, societies rely on people's behaviors to curb the spread of disease. To the extent that government-mandated actions are lacking or inconsistent, people's intrinsic motivation to comply with behavioral recommendations from public health officials becomes a vital determinant of disease spread. The United States (US), however, faced both cultural and socio-political barriers to gaining immediate and widespread changes in people's daily healthprotective behaviors. Research that investigates factors that shape people's intrinsic motivation to adopt behaviors to protect community health thus becomes essential. We hypothesize that high-quality social connections marked by coexperienced positive affect bear a unique association with prosocial tendencies that are tied to behaviors that reduce viral spread, such as handwashing, mask wearing, and social distancing. 


\section{Barriers to Coordinated and Widespread Behavior Change}

As an individualistic culture and a nation that values personal freedom over duty or security, the US faced cultural barriers to coordinated action at the onset of widespread community transmission of COVID-19 (Van Bavel et al., 2020). Lacking a unified federal response, state and local governments were left to make their own decisions about whether to implement "stay-at-home" orders and mandate face coverings. Resistance to new public policy (re: social distancing, mask wearing) appears typical in countries with "loose" government and social structures, in which high tolerance exists for deviations from social norms and rules (Gelfand et al., 2011; Van Bavel et al., 2020). Theory suggests that infectious diseases imposed selection pressures on human behavior, leading to the evolution of the behavioral immune system, a set of psychological mechanisms and social behaviors that serve as pathogen defense (e.g., ethnocentrism, conformity). These adaptations are less pronounced in individualistic cultures, which have less history of pathogen prevalence (Fincher et al., 2008; Schaller \& Park, 2011). Yet the US has stood out globally, even compared to its other Western (i.e., individualistic) counterparts, in its inability to control the spread of COVID-19 (Bremmer, 2020; Diamond \& Wheaton, 2020). Another barrier to successful containment is that the US COVID-19 response became increasingly politicized during a time of already high affective political polarization (Boxell et al., 2020). Affective political polarization, which refers to distrust and dislike of those from opposing political parties, posed a strong barrier to coordinated action, due to opposing groups holding different beliefs about the threat of COVID-19 and mask-wearing mandates (Igielnik, 2020; Moncus \& Connaughton, 2020; Van Bavel et al., 2020). Because pandemics can recur and widespread action and cooperation is essential to contain viral spread, it is imperative to understand social and psychological factors that support the enactment of behaviors that promote public health in cultures that are both "loose" and polarized.

\section{Co-experienced Positive Affect Builds Intrinsic Motives for Infection-Reducing Behaviors}

One way to get people to adopt infection-reducing behaviors is to increase their concern for others, so that other-oriented concern outweighs personal desires or perceived threats to cultural or socio-political values (Pfattheicher et al., 2020). We consider prosocial tendencies to represent a range of positive action-oriented dispositions, moral habits, and behaviors that transcend self-interest to promote or maintain communities. Prosocial tendencies are not fixed habits, but rather are social resources that may build up or erode over time. An offshoot of broaden-and-build theory (1998, for a review, see Fredrickson, 2013), the positivity resonance theory of co-experienced positive affect holds that positive affect collectively experienced between and among individuals can build such resources to a greater degree than positive affect that is experienced individually (Fredrickson, 2016). Specifically, a particular subtype of co-experienced positive affect, termed positivity resonance, occurs when two or more individuals show three key features across multiple emotionresponse systems: (a) shared positive affect (experiential), (b) caring nonverbal synchrony (behavioral), and (c) biological synchrony (physiological). These moments of high-quality social connection can emerge with the aid of perceived safety and real-time sensory connection between and among close friends, romantic partners, coworkers, acquaintances, or even complete strangers. The degree to which individuals experience or express positivity resonance or show biological synchrony during co-experienced positive affect has been found to predict levels of personal and interpersonal resources, such as mental health and relationship satisfaction, and does so independent of overall positive emotions and quantity of social interaction (Brown et al., in press; Chen et al., 2020; Major et al., 2018; Otero et al., 2019).

Individuals who frequently experience positivity resonance are also theorized to have accrued resources that transcend self-interest to promote other-oriented concern and behavior (i.e., prosocial tendencies). We posit that recurrent positivity resonance builds at least four interrelated prosocial tendencies, namely spirituality, empathy, altruism, and love of humanity, each of which is theoretically and/or empirically linked to features of positivity resonance. Spirituality, defined non-theistically as a feeling of interconnectedness and unity to all of life (Pargament et al., 2017; Piedmont, 1999), is known to be triggered by self-transcendent positive emotions (Saroglou et al., 2008; Van Cappellen et al., 2013). Empathy, defined as understanding and vicariously sharing another's emotions and feeling concern for the other in their time of suffering, is closely aligned with the mutual care feature of positivity resonance (Preston \& De Waal, 2002), and its development has been linked to warm and synchronous interactions between infants and their caregivers (Levy et al., 2019). Relatedly, altruism, defined as both the feeling of compassion and the actions of helping or giving (Lapsley \& Narvaez, 2014) can be produced between strangers simply through synchronous movements (Valdesolo \& DeSteno, 2011). Finally, we theorize that love of humanity, defined as positive feelings toward people in general coupled with beliefs that people are typically kind, good, and helpful (Campos et al., 2002; Sprecher \& Fehr, 2005), predisposes individuals to approach and be open to a range of interaction partners, which can subsequently reinforce positive feelings toward humanity in an upward spiral dynamic (e.g., Fredrickson \& Joiner, 2018). While each of these prosocial tendencies has 
been linked to one or more of the others in past research (e.g., spirituality, love of humanity, and altruism; Nai et al., 2018), the present work is the first to link these four specific prosocial tendencies to one another and to positivity resonance.

Our theory-driven prediction is that positivity resonance motivates infection-reducing behavior implicitly and indirectly, as mediated by a suite of prosocial tendencies. The present work thus complements and extends existing theory and research on the role of positive affect and nonconscious processes in health behavior maintenance (e.g., Custers \& Aarts, 2005; Van Cappellen et al., 2018). Whereas no published studies have yet examined the first link in this prediction (i.e., that from positivity resonance to prosocial tendencies), a number of published studies have supported the second link (i.e., from prosocial tendencies to infection-reducing behaviors). Empathy, for instance, has been investigated as a resource to increase health-related behaviors in pandemics and health care settings. Specifically, early in the COVID-19 pandemic, four studies (with samples from the US, UK, and Germany) found experimentally induced empathy for vulnerable populations to produce motivation to adhere to social distancing and mask wearing guidelines (Pfattheicher et al., 2020). Data gathered during past global health crises (i.e., SARS, West Nile) have also linked empathy to taking recommended health precautions (Lee-Baggley et al., 2004; Puterman et al., 2009). Plus, a prospective study of health care workers who received a 4-week empathy induction in their hospital ward (i.e., pre-tested posters of empathy-related photographs and psychoeducation on the benefits of empathy in patient interactions) found increased use of hand sanitizer relative to a control ward (Sassenrath et al., 2016). Beyond empathy, altruism has also been shown to motivate public health behaviors that protect others. Specifically, altruistic individuals are more likely to get vaccinated and be motivated by protecting others (Shim et al., 2012). Relatedly, educating people about herd immunity together with the social benefits of vaccination increases people's willingness to vaccinate (Betsch et al., 2013; Betsch et al., 2017). While we are not aware of research that links non-theistic spirituality and love of humanity with infection-reducing health behaviors, the studies on altruism and empathy support our prediction that prosocial tendencies motivate individuals to engage in behaviors that protect public health during epidemics and pandemics.

\section{The Current Research}

Consistent with the positivity resonance theory of coexperienced positive affect, we predict that individual differences in a set of prosocial tendencies (i.e., spirituality, empathy, altruism, love of humanity) will be associated with individual differences in the frequency of perceived positivity resonance. Moreover, we hypothesize that this set of prosocial tendencies will motivate the adoption of COVID-19 health behaviors (i.e., handwashing, social distancing, mask wearing) that function to protect community health. Specifically, our mediation hypothesis states that individuals with higher (vs. lower) perceived positivity resonance will engage in more (vs. less) COVID-19 health behaviors and that this association can be explained by higher (vs. lower) prosocial tendencies. We tested this mediation hypothesis both cross-sectionally and prospectively across two online studies. Study $1(N=1059)$ used the Day Reconstruction Method (DRM; Kahneman et al., 2004; Kahneman \& Krueger, 2006) with university and national samples to obtain episode-level reports of people's social interactions and emotions throughout a typical day during the height of the initial US outbreak of COVID-19, when stay-at-home orders (i.e., lockdowns) were widespread (i.e., in April 2020). A subset of the national sample was assessed in two additional follow-ups at 1 month and 3 months following the initial assessment. Study $2(N=227)$ offered a conceptual replication with a community sample while also testing whether daily reports of pre-pandemic perceived positivity resonance (from March 2019 to November 2019) predicted pandemic-era prosocial tendencies and COVID-19 health behaviors.

\section{Study 1}

\section{Method}

\section{Participants}

Participants come from two samples: a university sample (sample 1) and a national sample (sample 2). Sample 1 participants were recruited between March 28 and April 30, 2020, in exchange for course credit in psychology at either of two large public universities: one in California (CA) and the other in North Carolina (NC). During this time span, students at both universities had left campus and transitioned to remote learning due to the COVID-19 pandemic. To be eligible, students were over 18 and currently residing in the US. Initially, 595 students completed the survey. After removing participants who did not complete all study measures $(n=56)$, responses from internationally residing students $(n=10)$, participants who failed more than 1 of 3 attention checks $(n=34)$, and DRM responses that had too many overlapping episodes or repetitive, nonsensical episode names $(n=11)$, the final sample for analysis was $N=484(68 \%$ women, mean age $=$ $20.5, \mathrm{SD}=2.9$, range $=18-55$ years, $71 \%$ students attending the university in CA). Most participants identified as either Asian (36\%) or White or European American (34\%), whereas $16 \%$ identified as other, mixed, or preferred not to say, $11 \%$ identified as Hispanic or Latinx, and a small proportion identified as Black or African American (3\%).

Sample 2 participants were recruited through Amazon's Mechanical Turk (MTurk) during the first week of April 
2020 (April 1-8) for an initial assessment (time 1 (T1)). Each user received \$3 USD in exchange for participation. To be eligible, participants were over 18, fluent in English, and residing in the US. Initially, 725 participants consented to complete the survey. After removing those who failed more than 1 of 3 attention checks $(n=27), 698$ cases were assigned a personal ID number. Further cases were removed if they had missing or nonsensical entries for the DRM $(n=123)$, resulting in a T1 analysis sample of $N=575$ (50\% female, mean age $=36.7, \mathrm{SD}=11.5$, range $=18-74$ years $)$. A majority of participants identified as White or European American $(65 \%)$ with the next largest representation identifying as Black or African American (14\%). Additionally, $8 \%$ identified as Asian, $4 \%$ as Hispanic or Latinx, and $8 \%$ as other, mixed, or preferred not to say. While most participants were located anywhere in the US, CA $(n=167)$ and NC $(n=151)$ were oversampled to support possible location-dependent comparisons to the university sample. For education, a majority of the national sample had a bachelor's degree (51\%) or some college $(25 \%)$, whereas $18 \%$ held a post-graduate degree. Five percent indicated that a high school diploma was their highest education.

Of the 575 sample 2 participants in the $\mathrm{T} 1$ analysis sample, 307 returned for a follow-up survey approximately 1 month later (T2; April 29-May 7, 2020), and 285 returned for a second follow-up approximately 3 months from the initial assessment (T3; June 24-July 1, 2020). Returning respondents for T2 received an additional \$1 USD in exchange for participation in a shorter survey. Among these, we excluded 7 participants for either failing more than one attention check and/or having problems with their DRM responses: nonsensical text entries, no entries at all, or impossible or nonsensical time ranges, leaving a T2 sample of $N=300(n=91 \mathrm{CA}, n=$ $85 \mathrm{NC} ; 54 \%$ female, mean age $=39.2, \mathrm{SD}=12.0$, range $=19$ 74 years). The second follow-up at 3 months was available to anyone that completed the initial $\mathrm{T} 1$ assessment for an additional \$3 USD. Two participants were excluded for failing 2 attention checks, leaving a T3 sample of $N=283(n=87 \mathrm{CA}$, $n=68 \mathrm{NC} ; 52 \%$ female, mean age $=38.7, \mathrm{SD}=11.9$, range $=$ 19-74). Aside from the returning sample being significantly older, no other differences in demographic or primary variables of interest emerged between the sample that returned for T3 and those who completed T1 (see Table S1 in the Electronic Supplemental Materials).

\section{Materials and Procedures}

The Institutional Review Board of the University of North Carolina at Chapel Hill approved all procedures for the study (Study \# 20-0841). All participants provided informed consent for each survey. We used the DRM to assess participants' social interaction and emotions. The DRM minimizes reporting bias by cueing respondents to mentally visualize details of particular episodes in their day (e.g., "eating lunch," "phone call with sister"), rather than their general impression of themselves (Robinson \& Clore, 2002). Participants first reconstructed their previous day (i.e., "yesterday") by indicating what day of the week it was, then listing all episodes from the time they woke up until they went to bed. Participants were asked to give each reported episode a brief name, indicate its duration, and add any private notes to help recall their activities and experiences. After identifying their full set of episodes, for each episode (cued by its brief name), participants were asked whether they had been interacting with anyone for more than a few minutes, including by phone, text, email, or social media. For episodes that included a social interaction, participants were asked to report the proportion of time within that episode (from 0 to $100 \%$ ) they had been interacting and their mode of interaction: face-to-face, phone/ video (digital synchronous), mediated communication (e.g., email, texting; digital asynchronous), and/or not interacting. We then calculated the mean proportion of time spent in each mode of social interaction. Participants also indicated who they were interacting with: strangers/acquaintances, friend(s), family, romantic partner, and/or coworker. Participants could select multiple responses to account for the possibility that a given episode included multiple different social interaction types and/or partners.

For each social episode listed in the DRM, participants also reported on perceived positivity resonance (see Table 1). To reduce participant burden, we used two of the original seven items from the Perceived Positivity Resonance Scale (Major et al., 2018), selected to reflect the three key features of positivity resonance (i.e., co-experienced positivity, care, and synchrony). In a previous study that also assessed perceived positivity resonance at the episode level (i.e., initial assessments of study 2), this two-item scale demonstrated high correlation with the full seven-item scale $(r=.97$, Zhou et al., n.d.). We computed the mean of these two items per episode to create episode-level perceived positivity resonance scores. To create a person-level index, we computed the mean, within individuals, across all episode-level scores (social episodes only). For each episode (social and nonsocial), participants separately reported the greatest amount that they experienced any positive or negative emotion using a 5-point Likert-type scale ranging from 1 (not at all) to 5 (extremely). To create person-level indices, we computed mean positive and negative emotion scores, within individuals, across all episodes reported for the day. For the episodes in which participants reported interacting with others face-to-face for some proportion of time, they were asked "During this episode, how concerned were you that your activities could increase your risk of contracting or spreading the novel coronavirus," using a response scale of 1 (not concerned at all) to 7 (extremely concerned). Person-level indices were created by computing the mean within individuals across all episodes for the day. 


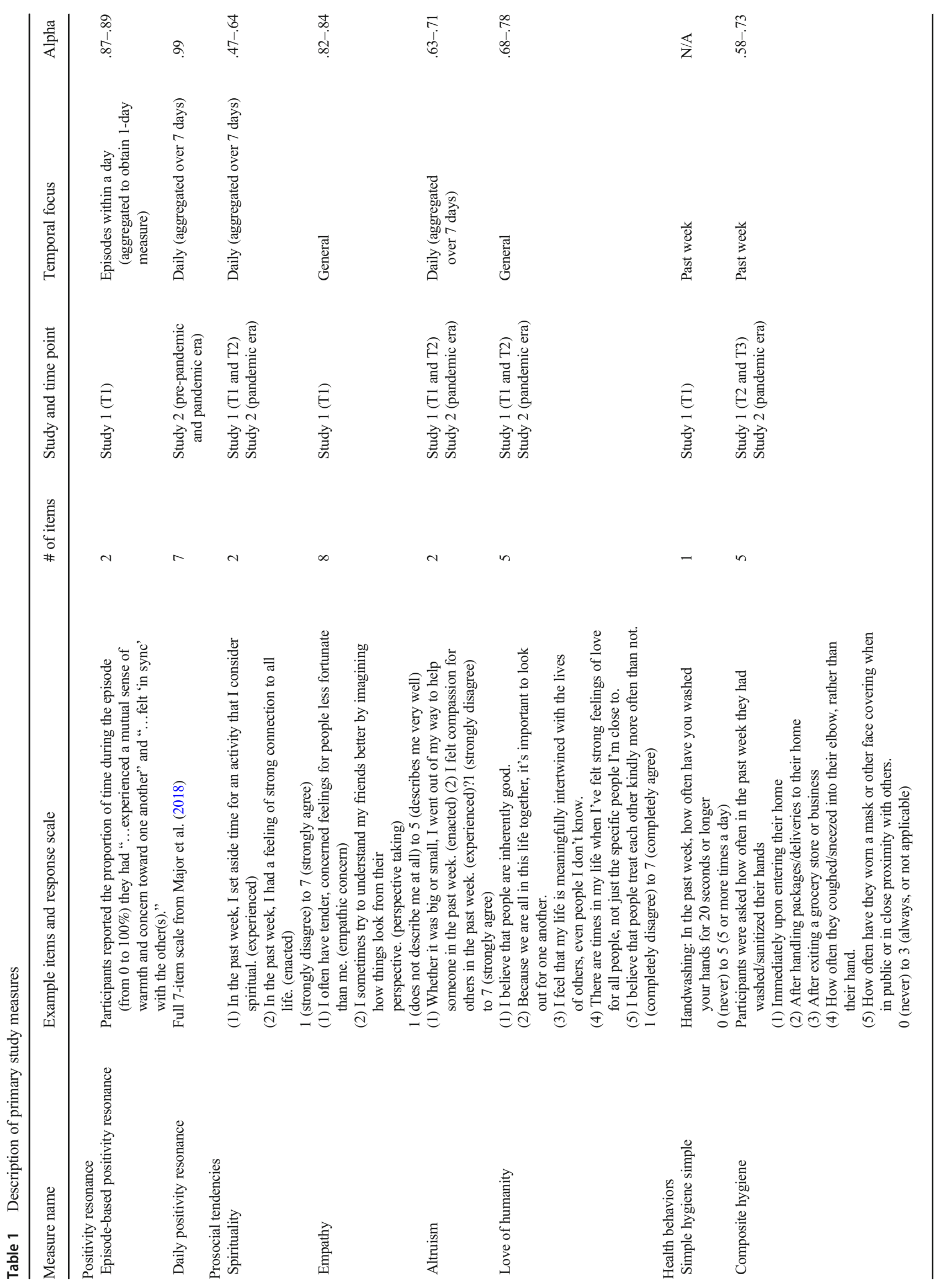


Prosocial tendencies were assessed at the individual level. Table 1 displays sample items and alpha ranges for both samples and all time points for all primary study measures. Participants responded to two items selected to assess both enacted and experienced spirituality (based on Underwood $\&$ Teresi, 2002, also used in study 2). To assess both emotional and cognitive empathy, participants completed the Empathic Concern and Perspective Taking subscales of the Brief Form of the Interpersonal Reactivity Index (B-IRI; Ingoglia et al., 2016). For altruism assessment, participants responded to two items selected to assess both enacted and experienced altruism (based on Rushton et al., 1981, also used in study 2). For love of humanity, items were selected from an unpublished "Different Kinds of Love" scale (Campos et al., 2002).

The initial assessment (sample 1 and $\mathrm{T} 1$ of sample 2) featured only a few single-item measures of COVID-19 health behaviors, whereas the 1-and 3-month follow-up assessments (T2 and T3; sample 2 only) included more fine-grained behavioral measures adapted from NSF-funded work by Shiota (2020). ${ }^{1}$ Table 1 displays health behavior items for all time points. For social distancing behavior, at T1, three 1-item measures were used to separately assess perceived social distancing effort, social distancing importance, and social contact outside the home. At T2 and T3, the measure of social distancing behaviors consisted of 5 items, again adapted from Shiota (2020), which were averaged for a composite social distancing score. To test the construct validity of our latent factor of prosocial tendencies, we also assessed COVID-19related charitable behavior. Specifically, the following oneitem question was used at T2: "There are many different ways to help others since the COVID-19 outbreak. Since the outbreak began in your area, have you engaged in any charitable behaviors? Below is an example of behaviors that are considered charitable: Gave money to someone in economic need, or to an organization. Gave another person food or household supplies they needed. Donated medical supplies such as masks and gloves. Contacted a person you know who may be lonely, to offer support. Helped another person with errands, such as groceries or prescriptions. Provided caregiving for someone outside your household who is sick. Volunteered with an organization providing coronavirus-related assistance." Participants responded from 0 (never) to 3 (3 or more times). This item was also adapted Shiota (2020).

We included additional items to serve as covariates. Political orientation was indexed as the mean of two items that captured both economic and social dimensions of political orientation (Carmines \& D'Amico, 2015): “When it comes to

\footnotetext{
${ }^{1}$ At the time of the initial assessment, face coverings had not been encouraged. The US CDC changed their recommended guidelines on mask wearing on April 3, 2020. Therefore, our second assessment included mask wearing, as well as other more detailed measures of hygiene practices.
} 
social issues, I am..." and "When it comes to economic issues, I am..." on a scale from 1 ("very liberal") to 7 ("very conservative"), with a midpoint of 4 ("moderate"; sample 1 alpha = .84 ; sample 2 alpha $=.93$ ). General concern for the self was measured with one item "I am concerned about COVID-19 because of the potential consequences (health or otherwise) for myself" on a scale of 1 (not concerned) to 7 (most concerning). We also assessed whether the participant or someone they live with was considered high-risk of severe illness from COVID-19 based on the CDC guidelines at the time of data collection. These guidelines were presented to participants (see Electronic Supplemental Materials S8 for full prompt), followed by the questions: "Are you or someone you live with considered high risk?" Participants could then check a box indicating themselves, someone they live with, or neither apply to me. Participants were also asked if they had been tested for COVID-19 with a yes or no response, and if they had been diagnosed with COVID-19 with a yes or no response. Additionally, participants were presented with a 13item illness symptoms checklist (Elliot \& Sheldon, 1998) and were asked how often they had experienced each symptom during the past week, from 1 (not at all) to 8 (very frequently). Illness symptoms were indexed by the mean of the 13 items ( sample 1 alpha $=.86$; sample 2 alpha $=.96)$.

\section{Analytical Approach}

Preliminary analyses were conducted to obtain descriptive statistics and zero-order correlations among study variables. Next, a series of multi-group structural equation models (SEMs) were conducted to test the hypothesized model across the populations represented by sample $1^{2}$ and sample 2 (Kline, 2015). The SEMs were fit in $\mathrm{R}$ with the package lavaan (Rosseel, 2012), with parameter estimates obtained with full information maximum likelihood estimator and unbiased errors obtained via bootstrapping with 1000 resamples (Nevitt $\&$ Hancock, 2001). The statistical significance of effect estimates was evaluated based on asymmetrical bootstrapped 95\% CIs (Bollen \& Stine, 1990), although for reference, we also report $p$ values, which correspond to the z-statistic under a standard normal distribution. Analyses occurred in two phases.

In phase 1 , we tested a measurement model to index prosocial tendencies as a latent variable using confirmatory factor analysis (CFA), specifically multi-group CFA (Kline, 2015) with samples 1 and 2 treated as separate groups. We hypothesized that a latent variable that indexed prosocial

\footnotetext{
$\overline{2}$ In preliminary analyses with the university sample, a multi-group CFA measurement model and a multi-group SEM path model for the two university groups (CA and $\mathrm{NC}$ ) demonstrated that the association among key variables of interest did not significantly differ across the two universities (see Table S2 in the Electronic Supplemental Materials). We thus combined participants from the two universities into one sample.
}

tendencies would emerge from the following four indicators: spirituality, empathy, altruism, and love of humanity (Fig. 1). All indicators were set to load on one latent factor (i.e., prosocial tendencies). Measurement invariance was investigated across the two samples. Because we were only interested in comparing covariation patterns across samples, we only aimed to establish metric invariance; that is, each indicator contributed to the latent factor to a similar degree across the two samples.

In phase 2, we integrated the hypothesized measurement model into the hypothesized path model (see Fig. 2). A direct path and an indirect path through prosocial tendencies from perceived positivity resonance to COVID-19 health behaviors were estimated. Integrating follow-up data from T2 and T3, we modeled the prospective mediational relationships among perceived positivity resonance, latent prosocial tendencies, and COVID-19 health behavior. To do so, we ran a separate path model with $\mathrm{T} 1$ positivity resonance as the predictor, $\mathrm{T} 2$ prosocial tendencies as the mediator, and T3 COVID-19 health behaviors as the outcome dependent variables.

For each of these phases, model specification, estimation, and selection were conducted in a stepwise manner. The initial models allowed all parameters to be freely estimated for each of the two samples. To acquire the best fitting and most parsimonious model, equality constraints were applied to the factor loadings and intercepts in the measurement model, with regression coefficients in path models and model fit statistics compared to the initial unrestricted model using likelihood ratio tests (Kline, 2015). Restrictions that did not significantly reduce model fit were retained. The final model fit was comprehensively evaluated using a combination of fit indicators including the comparative fit index (CFI), the root mean square error of approximation (RMSEA), and the standardized root mean square residual (SRMR). Conventional cutoff values for fit indices were used as a reference for judging models: CFI values greater than .95, RMSEA values less than .08 , and SRMR values less than .08 indicate good model fit (Schreiber et al., 2006).

In several secondary analyses, we tested alternatives to the hypothesized effects. Specifically, we reversed the implied direction of causal inference by positioning prosocial tendencies as the predictor and positivity resonance as the mediator in the mediation model. We also tested whether positivity resonance and prosocial tendencies have effects on shifts in COVID-19 behaviors across time. To do so, we set the outcome-dependent variables as residual change scores for health behaviors, obtained by predicting $\mathrm{T} 3$ behaviors controlling for $\mathrm{T} 2$ behaviors.

In subsequent sensitivity analyses, control covariates were added to the main models, including competing predictors of prosocial tendencies (i.e., positive emotions, negative emotions, and frequency of social episodes) and competing predictors of COVID-19 health behaviors (i.e., positive emotions, negative 
Fig. 1 Confirmatory factor analysis of prosocial tendencies. Standardized coefficient estimates are reported for both samples in a column of text, with the upper coefficients for sample 1 and the lower ones for sample 2 . Although the raw factor loadings were fixed to be equal across samples, the standardized factor loadings differed across samples as standardization was done within the sample. All factor loadings were significant $(p s<$ $.05)$

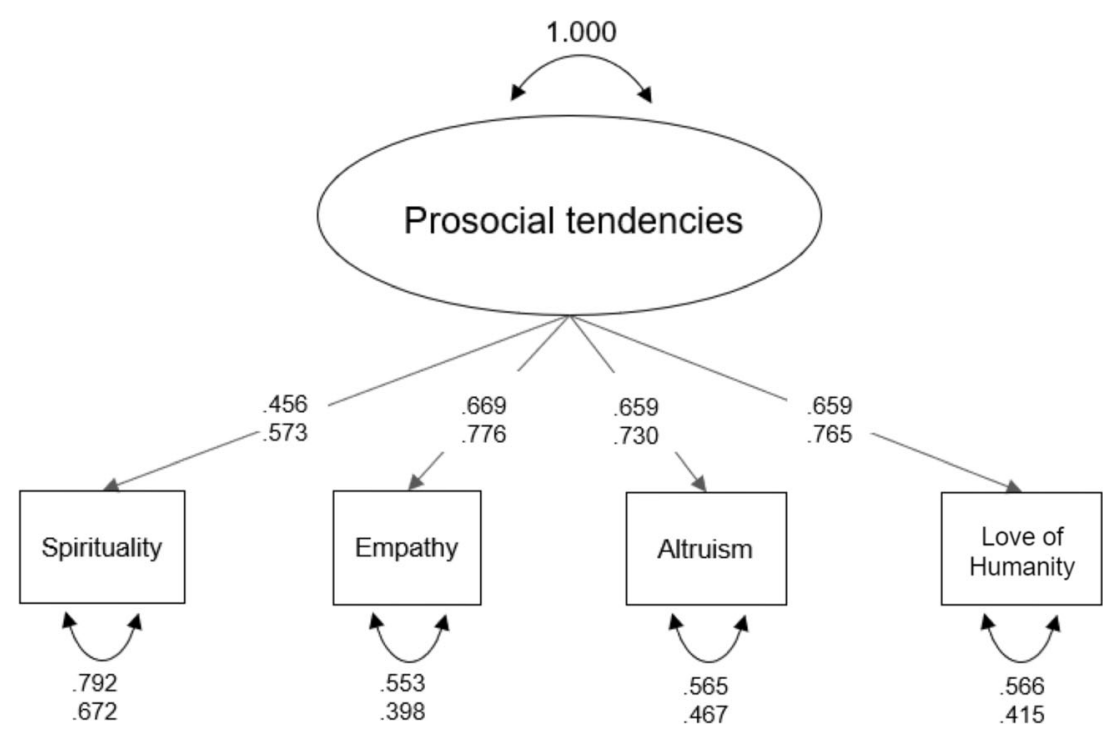

emotions, political orientation, COVID-19 self-concern, ethnicity, gender, age, education, illness symptoms, being considered high-risk of COVID-19, living with someone who is considered high-risk, tested for COVID-19, and diagnosed with COVID-19). To avoid overfitting with the large number of covariates, we followed the one parameter for every ten observations rule (Harrell et al., 1996) and kept the numbers of parameters less than or equal to 101 for cross-sectional models and 27 for prospective models. To do so, we separately tested two sets of covariates: (1) emotional and social covariates (positive emotions, negative emotions, and frequency of social episodes) and (2) demographic and COVID-19-related covariates (political orientation, COVID-19 self-concern, ethnicity, gender, age, education, illness symptoms, being considered high-

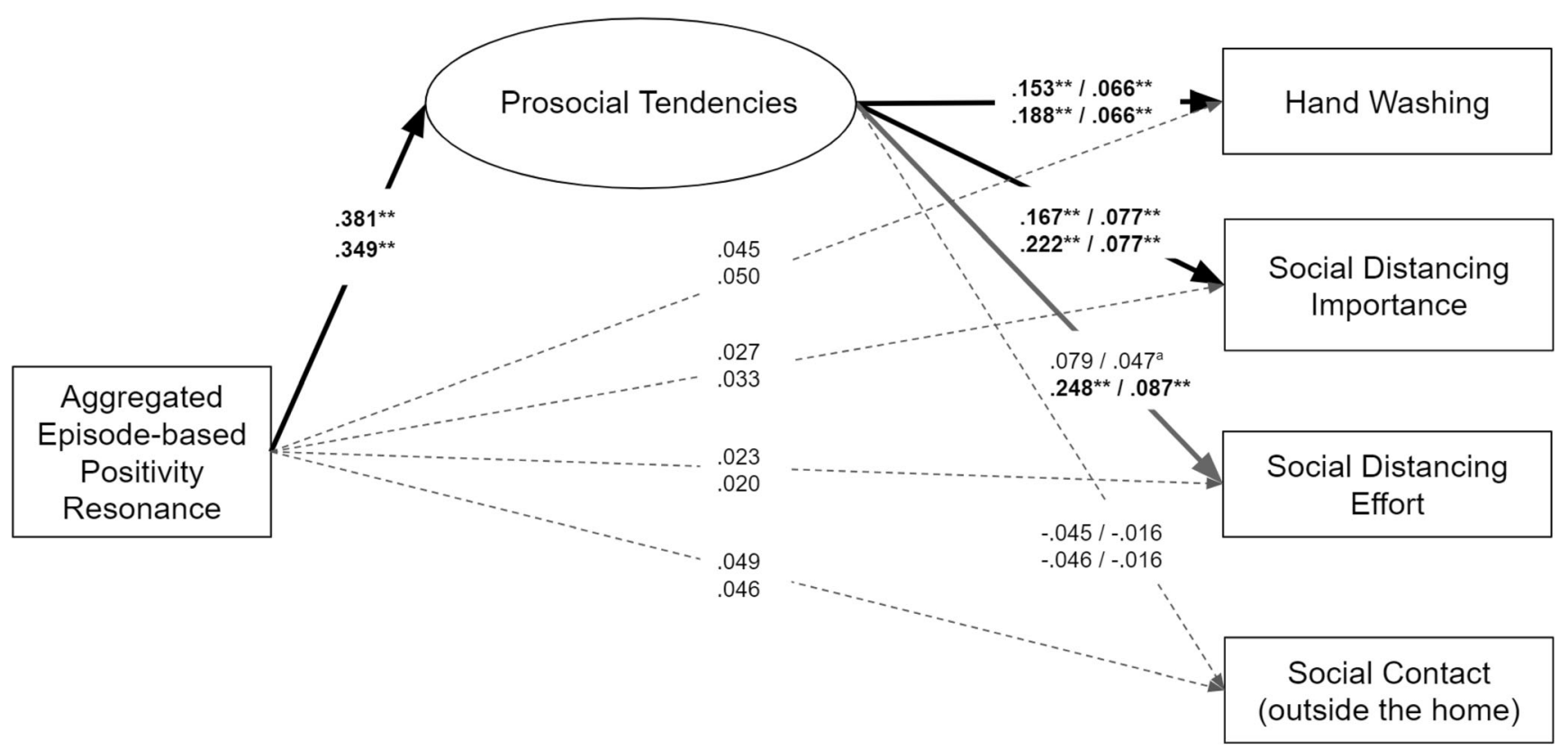

Fig. 2 Cross-sectional indirect effect of positivity resonance on T1 COVID-19-related behaviors through prosocial tendencies (study 1). Standardized coefficient estimates are reported for both samples in a column of text, with the upper coefficients for sample 1 and the lower ones for sample 2. Although the raw regression coefficients were fixed to be equal across the group for all paths except from prosocial tendencies to social distancing effort, standardized coefficient estimates differed as standardization was done within the sample. Coefficients before and after the forward slash (/) respectively show the direct effects of prosocial tendencies and the indirect effects of positivity resonance on behavior through prosocial tendencies. Unbroken and broken arrows respectively represent significant and insignificant paths. Superscript lowercase letter "a" indicates this path was only significant for sample 2 (MTurk sample) and not sample 1 (university sample). $* * p<.01$; key significant parameters in the mediation model are presented in boldface 
risk of COVID-19, living with someone who is considered high-risk, tested for COVID-19, and diagnosed with COVID19), as well as examined each behavioral outcome separately in models that contained the second set.

\section{Results}

\section{Preliminary Analyses}

A total of 10,728 episodes were reported through the DRM. Specifically, sample 1 reported a total of 4,482 episodes, with each participant reporting an average of 9.3 episodes (range $=$ 2-29 episodes). Sample 2 reported a total of 6,246 episodes at T1, with each participant reporting an average of 10.9 episodes (range $=1-30$ episodes).

Of these 10,728 total episodes, 5,286 were identified as involving a social interaction. Specifically, in sample 1,58\% of episodes $(2,611)$ were social, with each participant reporting an average of 5.4 episodes that included an interaction (range $=0$ 20 episodes). Of the 484 participants, 37 reported having only social episodes, 2 reported having only nonsocial episodes, and 20 reported only one social episode. In sample 2, 43\% of episodes $(2,675)$ was social, with each participant reporting an average of 4.7 episodes that included an interaction (range $=0-30$ episodes). Of the 575 participants, 28 reported having only social episodes, 39 reported having only nonsocial episodes, and 51 reported only one social episode.

Table 2 reports descriptive statistics for social interactions, perceived positivity resonance, and concern of spreading or contracting virus broken down by interaction partner and mode of communication for samples 1 and 2. Across the two samples, people reported that they spent most of their social time in face-to-face interactions, followed by digital synchronous or asynchronous interactions, and that most social partners were family and romantic partners, followed by friends, coworker/classmates, and then strangers. Consistent with previous research (Major et al., 2018), perceived positivity resonance was highest for face-to-face interactions. Participants also felt more frequent positivity resonance and were less concerned about contracting COVID-19 with romantic partners and family, followed by friends, coworkers, and strangers.

Tables 3 and 4 show the means, standard deviations, and zeroorder correlations among main study variables and covariates. Partially consistent with the primary hypothesis, at $\mathrm{T} 1$, prosocial tendencies were correlated with the 1-item assessments of handwashing, perceived importance of social distancing, and effort expended on social distancing, but not the number of days being in proximity with others outside one's home. For sample 2, $\mathrm{T} 1$ and $\mathrm{T} 2$ prosocial tendencies were also associated with $\mathrm{T} 2$ and $\mathrm{T} 3$ composite hygienic behaviors, but not T2 and T3 composite social distancing behaviors.

Demographic and COVID-19-related factors were also associated with COVID-19 health behaviors, and thus these factors were included in sensitivity analyses as control variables. At $\mathrm{T} 1$, one participant in sample 1 was diagnosed with COVID-19, among five who had been tested. In sample 2, also at T1, ten participants were diagnosed with COVID-19 out of 27 who had been tested. Cumulatively, in sample 2, fifteen had been diagnosed with COVID-19 out of 79 tested across $\mathrm{T} 1$ through $\mathrm{T} 3$.

\section{Multi-group Measurement Model of Prosocial Tendencies}

Table 5 reports the fit information of multi-group latent measurement models with four indicators (spirituality, empathy, altruism, and love of humanity) and of multi-group mediation models. The measurement model achieved our aim of metric

Table 2 Descriptive statistics on social interactions reported for samples 1 and 2 of study 1

\begin{tabular}{|c|c|c|c|c|c|c|}
\hline & \multicolumn{2}{|l|}{ Avg. \# of episodes } & \multicolumn{2}{|c|}{ Rescaled positivity resonance $(0-10)$} & \multicolumn{2}{|l|}{ Concern for virus } \\
\hline & Sample $1(N=484)$ & Sample $2(N=575)$ & $\mathrm{S} 1$ means $(\mathrm{SD})$ & $\mathrm{S} 2$ means $(\mathrm{SD})$ & $\mathrm{S} 1$ means $(\mathrm{SD})$ & $\mathrm{S} 2$ means (SD) \\
\hline \multicolumn{7}{|l|}{ Social target } \\
\hline Family & $3.2(n=385)$ & $3.4(n=407)$ & $5.96(2.43)$ & $7.02(2.40)$ & $1.47(.85)$ & $2.44(1.90)$ \\
\hline Partner & $3.4(n=171)$ & $3.4(n=254)$ & $6.71(2.35)$ & $7.62(2.13)$ & $1.69(.99)$ & $2.35(1.92)$ \\
\hline Friend & $3.1(n=404)$ & $2.4(n=311)$ & $5.71(2.45)$ & $6.62(2.34)$ & $1.64(1.14)$ & $2.98(2.20)$ \\
\hline Coworker & $1.7(n=131)$ & $2.3(n=231)$ & $4.34(2.56)$ & $5.27(2.51)$ & $2.27(1.72)$ & $3.35(2.17)$ \\
\hline Stranger & $1.7(n=212)$ & $1.9(n=219)$ & $3.86(2.57)$ & $4.65(2.76)$ & $2.77(2.00)$ & $3.99(2.24)$ \\
\hline \multicolumn{7}{|l|}{ Mode of interaction } \\
\hline Face to Face & $48 \%$ & $47 \%$ & $6.26(n=409)$ & $7.10(n=361)$ & & \\
\hline Digital synchronous & $30 \%$ & $24 \%$ & $5.82(n=307)$ & $6.38(n=202)$ & & \\
\hline Digital asynchronous & $22 \%$ & $29 \%$ & $4.75(n=264)$ & $5.39(n=262)$ & & \\
\hline
\end{tabular}

"Concern for virus" refers to the average level of concern for spreading or contracting COVID-19 (assessed only for face-to-face interactions). An uppercase $N$ indicates the total sample size, whereas the lowercase $n$ indicates the size of a subset of the sample who reported a specific type of interaction 


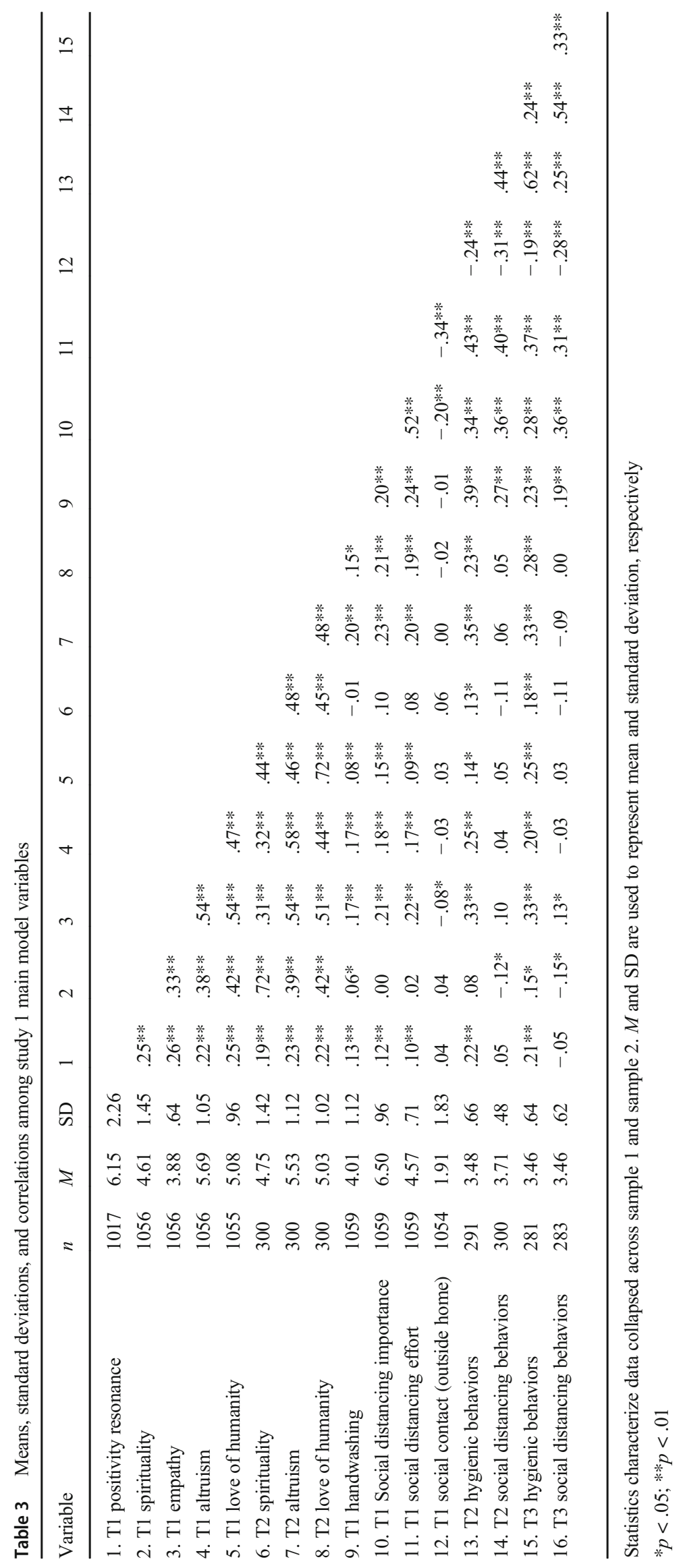




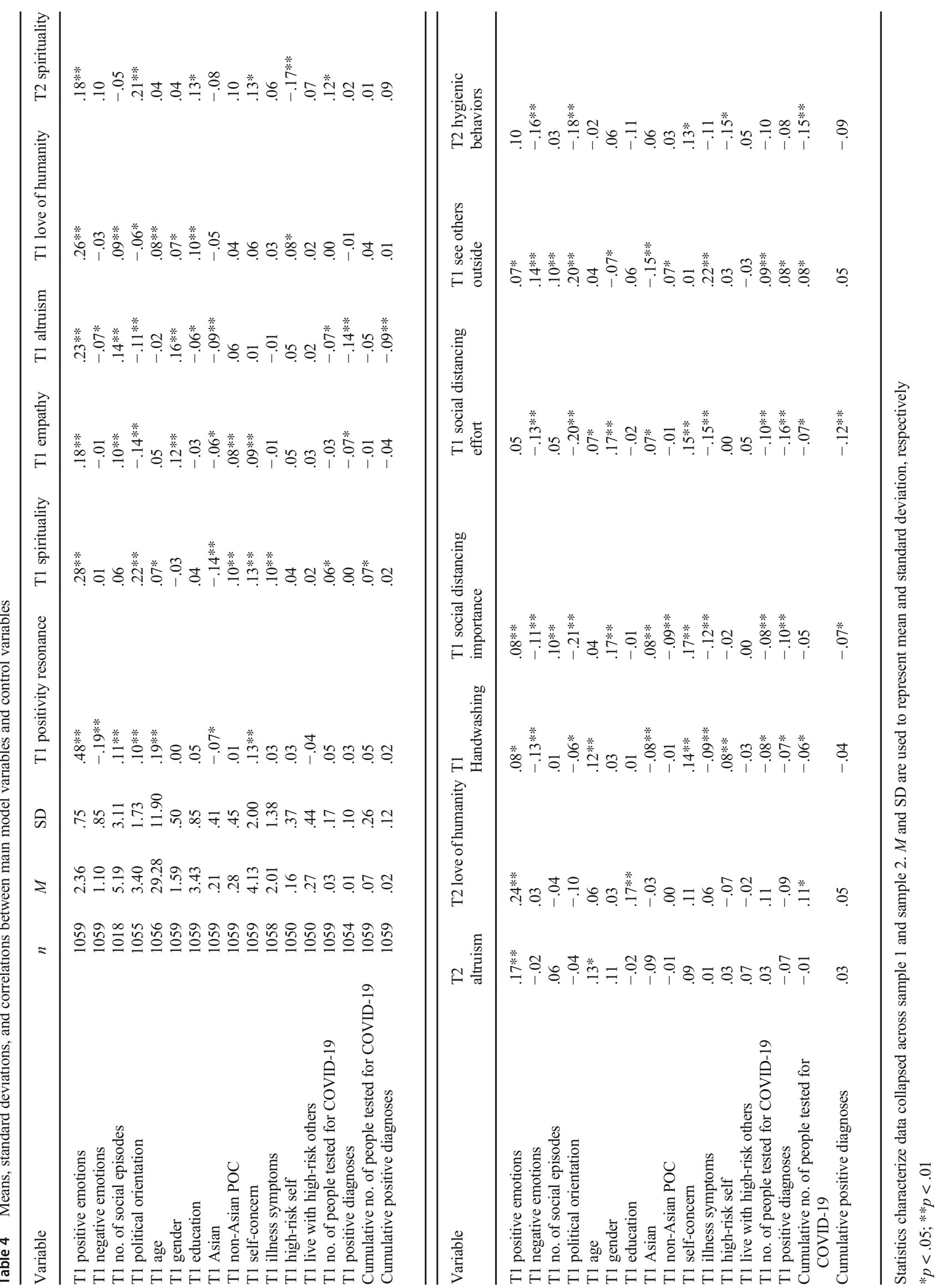


invariance, i.e., invariance in factor loadings of indicators on latent prosocial tendencies across the two samples. We note that scalar invariance was not achieved; i.e., the mean level of these indicators was not equal across the two samples. Yet because we were only interested in comparing covariation patterns across samples, metric invariance was sufficient. The metric invariance model demonstrated good fit $\left(\chi^{2}(7)=\right.$ 29.382, $p<.001, \mathrm{CFI}=.979$, RMSEA $=.078, \mathrm{SRMR}=.029$; Table 5$)$, and all indicators loaded significantly $(p \mathrm{~s}<.05)$ on latent prosocial tendencies (Fig. 1). Thus, we continued to incorporate the hypothesized latent factor for prosocial tendencies in subsequent path models.

As empathy was omitted by error from the $\mathrm{T} 2$ survey, we fit a separate measurement model with three indicators for $\mathrm{T} 1$ and $\mathrm{T} 2$ prosocial tendencies and exported the factor scores for all latent measurement models. Table S3 in the Electronic Supplemental Materials shows the means, standard deviations, and correlations between factor scores and charitable behaviors. The factor scores for a 4-indicator factor of $\mathrm{T} 1$ prosocial tendencies were extremely highly correlated with the 3-indicator factor of T1 prosocial tendencies $(r=.95)$ and highly correlated with the 3-indicator factor for T2 prosocial tendencies $(r=.74)$, the latter of which is on par with a good test-retest reliability threshold (Cicchetti, 1994). We thus consider the 3-indicator and 4-indicator latent measures of prosocial tendencies to be roughly equivalent. Next, we tested the construct validity of $\mathrm{T} 1$ and $\mathrm{T} 2$ latent factors of prosocial tendencies by correlating them with T2 and T3 COVID-19-related charitable behaviors. All associations were positive and statistically significant $(r=$ $.24-.33$; Table S3), thereby supporting the validity of the latent factor measurement.

\section{Cross-sectional Effects of Positivity Resonance on Time 1 COVID-19-Related Behaviors}

To select the best fitting multi-group model, we first fit a model that freely estimated the regression paths for each sample, before constraining these regression paths to the same value across samples. The constrained model showed a significant decrease in fit compared to the free model $\left(\Delta \chi^{2}(9)=22.384, p=008\right.$; Table 5). According to the parameter estimates in the free model, prosocial tendencies significantly predicted social distancing effort in sample 2 (national), but not in sample 1 (university). Therefore, we allowed the paths between prosocial tendencies and social distancing effort to be different in a subsequent, partially constrained model. This model has similar fit to the free model $\left(\Delta \chi^{2}(8)=12.769, p=.120\right.$; Table 5$)$. As the partially constrained model also showed good overall fit $\left(\chi^{2}(45)=112.501, p<.001, \mathrm{CFI}=.962\right.$, RMSEA $=$ .054 , SRMR $=.040$ ), we moved forward with it. Figure 2 shows the path diagram for this model. As hypothesized, aggregated perceived positivity resonance during social episodes predicted greater prosocial tendencies $(b=.108$, $95 \% \mathrm{CI}=[.86, .131], \beta_{\text {Sample } 1}=.381, \beta_{\text {Sample 2 }}=.349, p<$ $.001)$. Prosocial tendencies, in turn, predicted greater handwashing $\left(b=.282,95 \% \mathrm{CI}=[.146, .440], \beta_{\text {Sample } 1}\right.$ $\left.=.153, \beta_{\text {Sample 2 }}=.188, p<.001\right)$, and through prosocial tendencies, positivity resonance had a significant indirect effect on handwashing (indirect effect: $b=.030,95 \% \mathrm{CI}=$ $[.015, .049], \beta=.066, p=.001$; total effect: $b=.054,95 \%$ $\mathrm{CI}=[.026, .089], \beta=.116, p=.001)$. After controlling for the mediator (i.e., prosocial tendencies), positivity resonance no longer had a direct effect on handwashing ( $p=$ .175 ), suggesting complete mediation. The same complete mediation pattern was found for the perceived importance of social distancing: effect of prosocial tendencies: $b=$ $.275,95 \% \mathrm{CI}=[.158, .399], \beta_{\text {Sample } 1}=.167, \beta_{\text {Sample } 2}=$ $.222, p<.001$; indirect effect of positivity resonance: $b=$ $.030,95 \% \mathrm{CI}=[.017 .044], \beta=.077, p<.001$; and total effect: $b=.043,95 \% \mathrm{CI}=[.016, .069], \beta=.111, p=.001$. For perceived social distancing effort, this complete mediation pattern was significant only in the national sample (effect of prosocial tendencies: $b=.267,95 \% \mathrm{CI}=[.159$,

Table 5 Model fits of multi-group CFAs and SEMs

\begin{tabular}{|c|c|c|c|c|c|c|c|c|c|c|}
\hline & & $\chi^{2}$ & $d f$ & CFI & RMSEA & SRMR & Contrast & $\Delta \chi^{2}$ & $\Delta d f$ & $p$ \\
\hline \multicolumn{11}{|c|}{ CFA models } \\
\hline & Invariance type & & & & & & & & & \\
\hline 1 & Configural & 26.129 & 4 & .979 & .102 & .023 & - & - & - & - \\
\hline 2 & Metric & 29.382 & 7 & .979 & .078 & .029 & 2 vs. 1 & 3.253 & 3 & .354 \\
\hline 3 & Full scalar & 64.949 & 10 & .948 & .102 & .047 & 3 vs. 2 & 35.567 & 3 & $<.001$ \\
\hline \multicolumn{11}{|c|}{ Indirect effect models } \\
\hline & Constraint type & & & & & & & & & \\
\hline 1 & Freely estimate regression paths & 99.732 & 37 & .965 & .058 & .035 & - & - & - & - \\
\hline 2 & Complete equality constraint & 122.116 & 46 & .957 & .057 & .045 & 2 vs. 1 & 22.384 & 9 & .008 \\
\hline 3 & Partial equality constraint & 112.501 & 45 & .962 & .054 & .040 & 3 vs. 1 & 12.769 & 8 & .120 \\
\hline
\end{tabular}


.397], $\beta=.248, p<.001$; indirect effect: $b=.029,95 \%$ CI $=[.017, .046], \beta=.087, p<.001)$, whereas no significant effects on social distancing effort emerged in the university sample ( $p s \geq .150$ ). Days spent in proximity to people outside home, however, were neither predicted by prosocial tendencies nor predicted by positivity resonance $(p s>.185)$.

\section{Prospective Effects of Positivity Resonance on COVID-19-Related Behaviors}

In a separate model, we examined the effects of positivity resonance on future COVID-19 health behaviors through prosocial tendencies (sample 2 only). Figure 3 shows the path diagram for this model. The model showed excellent overall fit $\left(\chi^{2}(6)=7.122, p=.310, \mathrm{CFI}=.994\right.$, RMSEA $=.026$, SRMR $=.027) . \mathrm{T} 1$ positivity resonance predicted $\mathrm{T} 2$ prosocial tendencies $(b=.128,95 \% \mathrm{CI}=[.058, .196], \beta=$ $.338, p<.001)$, which, in turn, predicted T3 hygienic behaviors $(b=.305,95 \% \mathrm{CI}=[.134, .508], \beta=.437, p=.002)$. T1 positivity resonance also had an indirect effect mediated by $\mathrm{T} 2$ prosocial tendencies on $\mathrm{T} 3$ hygienic behaviors (indirect effect: $b=.039,95 \% \mathrm{CI}=[.016, .080], \beta=.148, p=.011$; total effect: $b=.056,95 \% \mathrm{CI}=[.023, .093], \beta=.212, p=.002)$ and no direct effect after controlling for the mediator $(p=$ .416), suggesting full mediation.
This mediational pattern was not significant for $\mathrm{T} 3$ social distancing: effect of prosocial tendencies: $b=-.052,95 \% \mathrm{CI}$ $=[-.171, .062], \beta=-.078, p=.392 ;$ indirect effect: $b=$ $-.007,95 \% \mathrm{CI}=[-.026, .007], \beta=-.026, p=.405$; and total effect: $b=-.011,95 \% \mathrm{CI}=[-.038, .023], \beta=-.045, p$ $=.452$. Whereas the link between prosocial tendencies and social distancing effort and behaviors was mixed in the previous cross-sectional analyses, the prospective findings for the national sample suggest that prosocial tendencies were unrelated to these behaviors.

\section{Secondary Analyses}

In secondary analyses, we first tested alternative mediation models, for both the cross-sectional and prospective analyses, in which positivity resonance was positioned as the mediator that links prosocial tendencies, as predictor, to the outcomes of health behaviors. No significant indirect effects of prosocial tendencies through positivity resonance emerged in these alternative models (see Table S4 in the Electronic Supplemental Materials). Second, we also tested the indirect effect of positivity resonance through prosocial tendencies on shifts in health behaviors, which were operationalized as residual changes in outcome behaviors (i.e., each of $\mathrm{T} 3$ behaviors was regressed on their respective $\mathrm{T} 2$ behaviors). This model revealed that prosocial tendencies significantly predicted shifts in hygienic behaviors $(b=.205$,

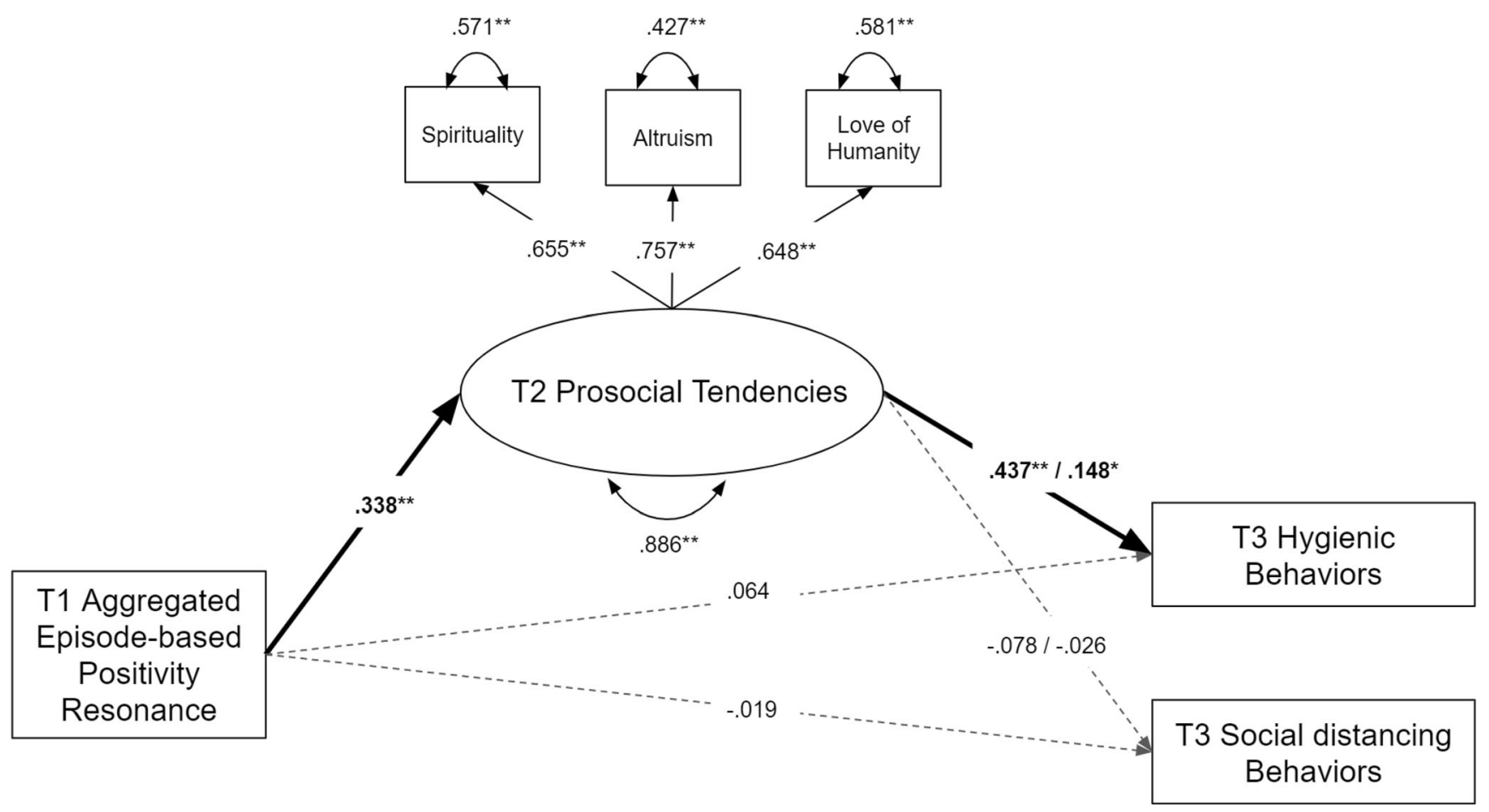

Fig. 3 Longitudinal indirect effect of $\mathrm{T} 1$ positivity resonance on $\mathrm{T} 3$ followup COVID-19-related behaviors through T2 prosocial tendencies (study 1). Standardized coefficient estimates are reported. Coefficients before and after the forward slash (/) respectively show the direct effects of prosocial tendencies and the indirect effects of positivity resonance on behavior through prosocial tendencies. Unbroken and broken arrows respectively represent significant and insignificant paths. ${ }^{*} p \leq .01$; key significant parameters in the mediation model are presented in boldface 
$95 \% \mathrm{CI}=[.036, .383], \beta=.234, p=.019)$. Positivity resonance also had a significant total effect on shifts in hygienic behaviors $(b=.031,95 \% \mathrm{CI}=[.004, .064], \beta=.123, p=.040)$, which was completely mediated by prosocial tendencies (indirect effect: $b=$ $.019,95 \% \mathrm{CI}=[.003, .048], \beta=.076, p=.090$; direct effect: $b=$ $.012,95 \% \mathrm{CI}=[-.021, .046], \beta=.046, p=.488)$. No significant effects emerged regarding shifts in social distancing behaviors (ps > .214).

To test whether positivity resonance predicted prosocial tendencies independent of experienced emotions and quantity of social interaction, we regressed prosocial tendencies on positive and negative emotions, and interaction frequency (number of social episodes); we also regressed behavioral outcomes on positive and negative emotions. The significant pattern of crosssectional and prospective findings remained mostly unchanged after controlling for these covariates (see Table S5 in the Electronic Supplemental Materials). The one exception was that for the university sample, the cross-sectional indirect link between positivity resonance and perceived social distancing effort through prosocial tendencies became significant after the covariates were introduced (effect of prosocial tendencies: $b=.132$, $95 \% \mathrm{CI}=[.025, .258], \beta=.133, p=.026$; indirect effect: $b=$ $.010,95 \% \mathrm{CI}=[.002, .020], \beta=.032, p=.031)$.

In separate models, we tested the effects of prosocial tendencies and indirect effects of positivity resonance on individual COVID-19-related behaviors while controlling for demographic and COVID-19-related covariates (i.e., age, gender, ethnicity, political orientation, education, illness symptoms, being considered high-risk of COVID-19, living with someone who is considered high-risk, tested for COVID-19, and diagnosed with COVID-19). For crosssectional models, we controlled for $\mathrm{T} 1$ test and diagnosis status, whereas for prospective models, we controlled for cumulative test and diagnosis status. After taking into account these factors, the pattern of cross-sectional and prospective findings did not meaningfully change (see Table S6 in the Electronic Supplemental Materials).

\section{Strength and Limitations of Empirical Approach}

One strength of study 1 is the latent variable approach to assess the broad construct of prosocial tendencies. This approach reduced the impact of low reliability in the measures of any single indicator variables. A second strength is the use of the DRM, which assessed experiences aggregated across all episodes on a typical day for each respondent during the early stages of the pandemic. A third strength is the use of COVID-19 health behavior measures across multiple time points, which allowed us to examine cross-sectional and prospective effects on behaviors as well as effects on behavior shifts. One limitation of study 1 is that it measured positivity resonance at a time when most in the US were under stay-at-home orders, which likely altered the quantity, quality, and modes of respondents' typical social interactions, perhaps differently so across individuals. This approach does not capture how people interact during non-pandemic times and may have restricted the range of people's social and emotional experiences. We undertook study 2 both to conceptually replicate the findings of study 1 and to address this limitation using data collected prior to the COVID-19 pandemic. In study 2, people's day-to-day social connections were assessed in 2019. Our primary aim was to examine how participants' characteristic levels of positivity resonance, pre-pandemic, prospectively predicted their prosocial tendencies during the 2020 pandemic and, indirectly, their COVID-19 health behaviors.

\section{Study 2}

\section{Method}

\section{Participants}

Participants $(N=405)$ from an initial 2019 NC communitybased daily diary study (that included randomization to one of 4 study conditions) were recontacted via email and asked to participate in a weeklong follow-up assessment in April 2020, while under state-issued stay-at-home orders, with the opportunity to earn a $\$ 25$ gift card. All who consented were used in the analysis $(N=227 ; 82 \%$ female, mean age $=34.5, \mathrm{SD}=11.4$, range $=20-64$ years). In this follow-up sample, $71 \%$ identified as White, $12 \%$ as Black or African American, $8 \%$ as Asian, and $6 \%$ as Hispanic. A large portion of the sample had a bachelor's degree $(42 \%)$ or a master's degree $(33 \%)$, and $11 \%$ had a professional or doctoral degree, $4 \%$ an associate's degree, $7 \%$ some college, and $2 \%$ a high school degree or less. The returning participants did not differ from the non-returning ones in demographic variables, condition assignment, or perceived positivity resonance (see Table S7 in the Electronic Supplemental Materials)

\section{Materials and Procedures}

The Institutional Review Board of the University of North Carolina at Chapel Hill approved all procedures for the study (Study \# 18-2810). The community sample had previously participated, on a rolling basis, in a 5-week social connection study between March and November of 2019. The primary objective of that pre-registered study was to test whether positivity resonance can increase prosocial tendencies (results reported in Zhou et al., n.d.), and participants were randomized into experimental and control conditions accordingly. Participants had completed, over 5 weeks, nightly reports on their emotions, select social interactions, and prosocial tendencies, both enacted and experienced. To minimize the impact of experimental condition and maximize the sample size, the current study 2 drew from each participant's first week of 
nightly reports during their 2019 study involvement. ${ }^{3}$ Between April 28 and May 11, 2020, after providing informed consent, follow-up participants completed nightly surveys for 7 days to assess perceived positivity resonance during social interactions (with close vs. distal others, assessed separately) and two prosocial tendencies (spirituality and altruism). Daily items were averaged for the week for analyses. COVID-19 health behaviors and one prosocial tendency (love of humanity) were reported once in reference to the past week after the 7-day reporting period (see Table 1). Prosocial tendencies were assessed with the same scales as used in study 1 , except we inadvertently did not measure empathy in study 2 . Charitable COVID-19 behaviors (1 item) were also measured the same way as study 1 to validate the prosocial latent factor in this independent sample.

Pre-pandemic positivity resonance was assessed by aggregating the first 7 days of nightly reports from the 2019 study (see Table 1). Each evening (after 6 p.m. local time), participants were asked: "Think back to the single longest interaction you had with one or more close others [strangers or acquaintances] today. Take a moment to recall and mentally relive this event, including how the event unfolded, what time of day it was, and what it was like." Next, positivity resonance was captured using the full 7-item Perceived Positivity Resonance Scale for each target (Major et al., 2018). For the current study, both targets (close and distal) were averaged together for a total perceived positivity resonance score. Pandemic-era positivity resonance was assessed daily using this same 7-item measure across the seven days of the followup survey.

Besides aforementioned demographic factors, we also include pre-pandemic daily positive and negative emotion in the sensitivity analysis model as controls. Pre-pandemic daily emotions were assessed by aggregating the same first 7 days of nightly reports from the 2019 study that were used to index pre-pandemic positivity resonance. Emotions were assessed using the same daily 1-item measures (one for positive and one for negative emotion) as in study 1 . We measured political orientation on day 7 (i.e., the final day of the follow-up assessment in 2020) using the same two items as used in study 1 ( $\alpha=.85$ ). Same as study 1 , participants reported if they or someone they lived with was considered high-risk of COVID19 and if they had been tested or diagnosed with COVID-19 (five people were tested, but no one was diagnosed as positive in this sample). Illness symptoms were indexed by a single item "Today I felt physically unwell or under the weather" (1 $=$ not at all to $5=$ extremely), which was completed daily and averaged for the same follow-up week as reporting on

\footnotetext{
${ }^{3}$ The focus of the current study 2 is not on the effects of randomized conditions. Even so, condition served as a control variable in sensitivity analyses. The experimental manipulation occurred during the first week of nightly reports.
}

pandemic-era positivity resonance, prosocial tendencies, and health behaviors.

\section{Analytical Approach}

Similar to study 1, descriptive statistics and zero-order correlations among the study variables were computed in preliminary analyses. Next, we used SEM to test path models using the same estimation approach and software. A common latent factor of prosocial tendencies was extracted from the three available indicators (spirituality, altruism, and love of humanity) using CFA. As this measurement model was fully saturated with three indicators, we could not evaluate fit prior to including it in the structural model. We then estimated a direct path and an indirect path through prosocial tendencies from positivity resonance (measured pre-pandemic and during the pandemic) to COVID-19-related behaviors (Fig. 4). A biascorrected bootstrapping procedure (1,000 resamples) was used to obtain $95 \%$ confidence interval.

In sensitivity analyses, to control for differences in the time of pre-pandemic positivity resonance assessments, we added an interaction term to the path model, crossing perceived positivity resonance with the number of days passed between the last assessment in 2019 and the follow-up survey in 2020. Control covariates were also added to the path analysis, including competing predictors of prosocial tendencies (experimental condition, positive emotions, negative emotions) ${ }^{4}$ and competing predictors of COVID-19-related behavior (experimental condition, positive emotions, negative emotions, political orientation, feeling under the weather, being considered high-risk of COVID-19, living with someone who is considered high-risk, and tested for COVID-19). To avoid overfitting, we followed the one parameter for every ten observations rule (Harrell et al., 1996) and kept the numbers of parameters less than or equal to 22 . To do so, we tested two sets of covariates separately: (1) experimental conditions and emotion covariates and (2) demographic and COVID-19-related covariates (political orientation, ethnicity, gender, age, education, feeling under the weather, being considered highrisk of COVID-19, living with someone who is considered high-risk, and tested for COVID-19), while examining each behavioral outcome in separate models. We assessed model fit similar to study 1, using a combination of fit indicators (CFI, RMSEA, and SRMR).

\section{Results}

\section{Preliminary Analyses}

Table 6 shows the means, standard deviations, and zero-order correlations among included variables. Partially consistent

\footnotetext{
${ }^{4}$ Quantity of social interaction was assessed in study 1 but not study 2.
} 
with the primary hypotheses, latent prosocial tendencies were associated with hygienic behaviors. Associations between demographic variables and COVID-19-related behavior also emerged, and therefore, these variables were controlled in sensitivity analyses. Perceived positivity resonance slightly increased from the pre-pandemic to pandemic era $(t(226)=$ 2.94, $p=.004, d=.20$ ).

Replicating findings of study 1 , the latent factor of prosocial tendencies was associated with charitable behaviors $(r=.29, p<.01)$, providing evidence for the construct validity of our latent factor measure.

\section{Cross-sectional Effects of Pandemic-Era Positivity Resonance on COVID-19-Related Behaviors}

Figure 4 shows the model results for both pre-pandemic and pandemic-era positivity resonance. The cross-sectional model using pandemic-era positivity resonance shows excellent fit $\left(\chi^{2}(6)=7.371, p=.288, \mathrm{CFI}=.993, \mathrm{RSMEA}=.032\right.$, SRMR $=.023)$, and the overall pattern of significant findings replicated those from study 1 (Fig. 4). Because pandemic-era positivity resonance was measured around the same time as prosocial tendencies, we also tested an alternative mediation model in which prosocial tendencies is positioned as the predictor and positivity resonance as the mediator. Similar to study 1 , positivity resonance did not mediate the association between prosocial tendencies and health behaviors (see Table S4 in the Electronic Supplemental Materials).

\section{Prospective Effects of Pre-pandemic Positivity Resonance on COVID-19-Related Behaviors}

The hypothesized longitudinal mediation model for prepandemic positivity resonance (Fig. 4) showed excellent fit $\left(\chi^{2}(7)=3.852, p=.797, \mathrm{CFI}=1.000\right.$, RSMEA $\left.=.000\right)$. Pre-pandemic positivity resonance predicted greater pandemic-era prosocial tendencies $(b=.166,95 \% \mathrm{CI}=$ $[.099, .271], \beta=.501, p<.001)$ and, through prosocial tendencies, indirectly predicted more frequent hygienic behaviors (effect of prosocial tendencies: $b=.298,95 \% \mathrm{CI}=[.098$, $.585], \beta=.258, p=.009$; indirect effect of positivity resonance: $b=.050,95 \% \mathrm{CI}=[.019, .093], \beta=.129, p=.008)$. The direct effect of positivity resonance was nonsignificant after controlling for prosocial tendencies, suggesting complete meditation $(b=.014,95 \% \mathrm{CI}=[-.037, .061], \beta=.035, p=$ $.595)$. Similar to study 1 , social distancing behaviors were neither related to prosocial tendencies $(b=.002,95 \% \mathrm{CI}=$ $[-.185, .174], \beta=.002, p=.980)$ nor predicted by prepandemic positivity resonance (direct effect: $b=.014,95 \%$ $\mathrm{CI}=[-.037, .061], \beta=.043, p=.595$; indirect effect: $b=$

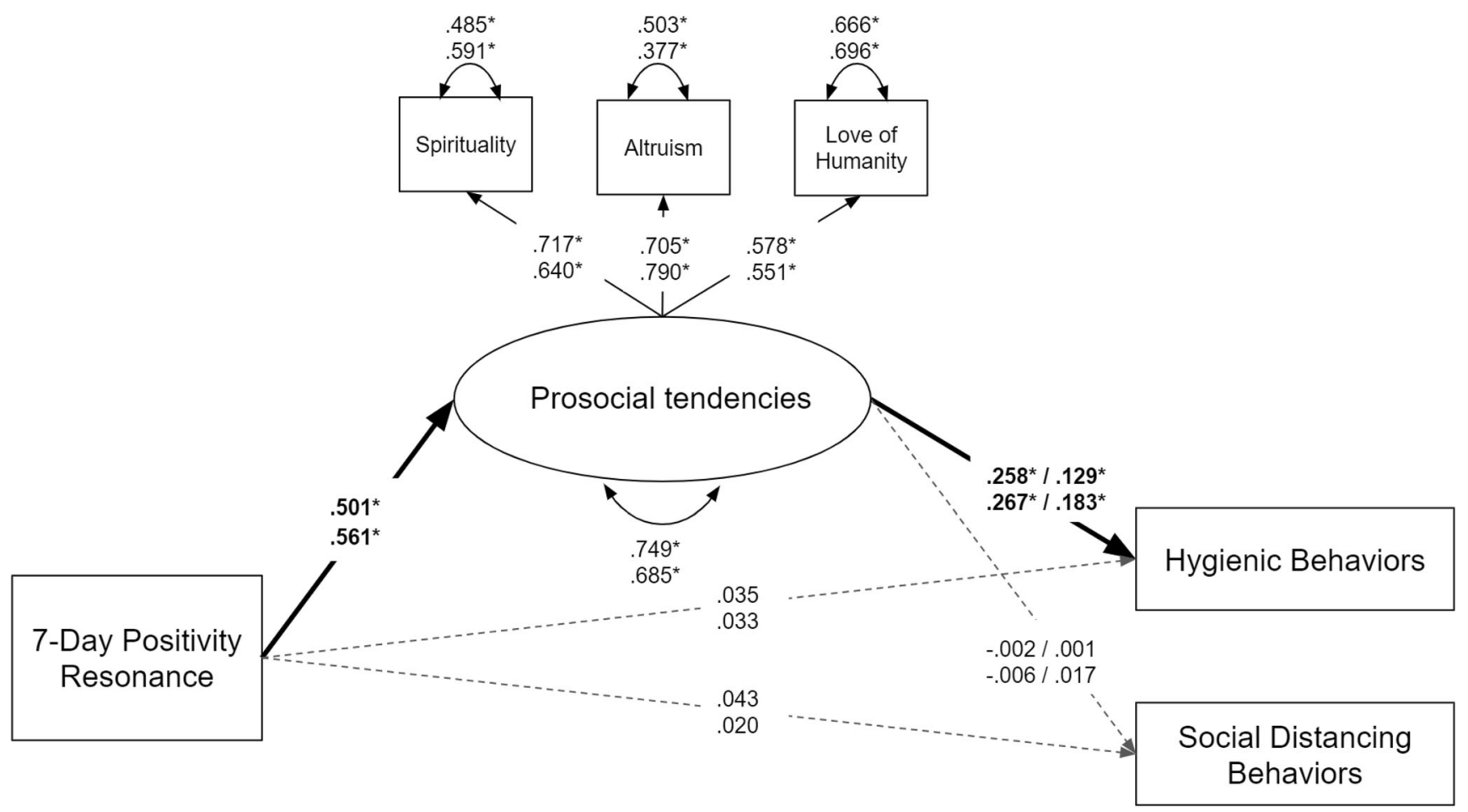

Fig. 4 Replication of hypothesized indirect effects using cross-sectional and longitudinal data from study 2. Pre-pandemic positivity resonance and pandemic-era positivity resonance were tested in separate models. The upper and the lower standardized coefficients are for models that respectively analyzed pre-pandemic and pandemic-era positivity resonance. Coefficients before and after the forward slash (/)

respectively show the direct effects of prosocial tendencies and the indirect effects of positivity resonance on behavior through prosocial tendencies. Unbroken and broken arrows respectively represent significant and insignificant paths. $* p \leq .01$; key significant parameters in the mediation model are presented in boldface 


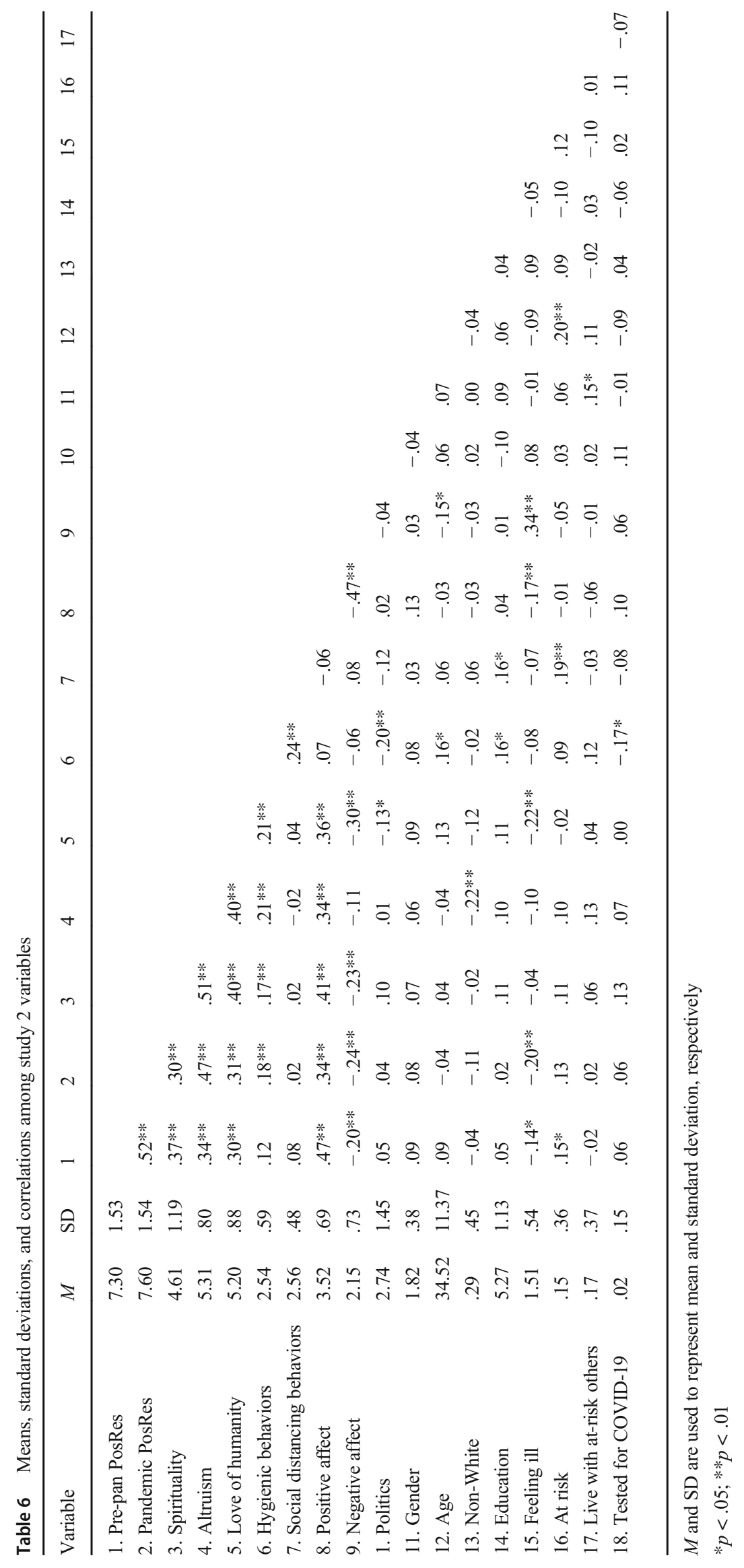


$.000,95 \% \mathrm{CI}=[-.030, .032], \beta=.001, p=.980$; total effect: $b=.014,95 \% \mathrm{CI}=[-.023, .048], \beta=.037, p=.439)$.

\section{Sensitivity Analyses}

We tested whether the effect of pre-pandemic positivity resonance on pandemic-era prosocial tendencies differed by how much time had passed between the pre-pandemic assessment and the follow-up COVID-19-related survey. The interaction effect was not significant $(b=.000, \mathrm{SE}=.001,95 \% \mathrm{CI}=$ $[-.001, .002], p=.938)$. Experimental condition also did not influence pre-pandemic positivity resonance, assessed during week 1 of the intervention $(F(3,223)=1.396, p=$ $.245)$, nor pandemic-era positivity resonance $(F(3,223)=$ $1.398, p=.244)$.

To test whether pre-pandemic positivity resonance predicted prosocial tendencies independent of other factors, we regressed prosocial tendencies on positive emotions, negative emotions, and experimental conditions. In predicting COVID19-related health behaviors, we also controlled for experimental condition. Introducing these covariates did not change the patterns of findings for hygienic behaviors and social distancing behaviors (see Table S8 in the Electronic Supplemental Materials).

Separately, we tested whether the effects of positivity resonance and prosocial tendencies on COVID-19 health behaviors remain after controlling for demographic and COVID19-related covariates (age, gender, ethnicity, political orientation, education, being considered high-risk, living with someone considered high-risk, and tested for COVID-19). After covariates were introduced, the pattern of results remained the same for hygienic behaviors and social distancing behaviors (see Table S9 in the Electronic Supplemental Materials). Thus, as for study 1 , the indirect effect of positivity resonance on COVID-19 health behavior emerged prospectively and was independent from any effects of positive or negative emotions or experimental conditions, or effects of demographic and COVID-19-related factors.

\section{Strength and Limitations of Empirical Approach}

Study 2 overcame a key limitation of study 1 by assessing perceived positivity resonance in 2019 , prior to the onset of the COVID-19 pandemic. Moreover, we measured perceived positivity resonance in study 2 with the full 7-item scale (Major et al., 2018), across two categories of social partners (close vs. distal others), each evening for a week. This assessment of positivity resonance should thus be more stable against day-to-day fluctuations and unaffected by the substantial changes in people's social opportunities created by stayat-home mandates. These differences in assessment strategy increase the generalizability of the conceptual replication offered by study 2 .

\section{Discussion}

Evidence from two studies (total $N=1,286$ ) support the hypothesis drawn from the positivity resonance theory of coexperienced positive affect that social interactions marked by mutual warmth, concern, and synchrony are associated, both cross-sectionally and prospectively, with reported pandemicera health behaviors that can curb viral spread (i.e., washing hands and wearing masks). For an illustration, in the national sample of study 1, among people who scored in the top $20 \%$ on positivity resonance, $87 \%$ reported "almost always or always" practicing hygienic behaviors, such as handwashing and mask wearing, whereas among the bottom $20 \%$, only $56 \%$ reported doing the same. Furthermore, both studies suggest that the pathway between positivity resonance and COVID-19 health behavior is indirect, as hypothesized, accounting for by the higher levels of prosocial tendencies associated with more frequent experiences of positivity resonance. Additionally, our latent measure of prosocial tendencies in both studies was predictive of reporting more COVID-19related charitable acts, providing validity for this mediating construct. While the present study focuses on individual differences rather than behavioral adoption, limited evidence also emerged in study 1 that these effects grew stronger over time. Specifically, positivity resonance predicted significant increases in COVID-19 health behaviors across time points, indirectly through prosocial tendencies, providing ample avenues for future research on effective public health interventions.

Empirical support for the behavior of social distancing was mixed. In study 1 , for both samples, perceived positivity resonance indirectly predicted the importance people reported placing on social distancing. The effort they reported putting into social distancing, however, was only predicted (indirectly) by positivity resonance in the national sample, and then only cross-sectionally using a 1-item index, and not longitudinally, using a composite index. Additionally, no effects for the composite index of social distancing emerged in study 2.

Evidence for mediation through prosocial tendencies remained significant after accounting for other competing predictors of prosocial tendencies and health behaviors. In regard to competing predictors of prosocial tendencies, the association between positivity resonance and COVID-19 health behaviors was held after accounting for overall positive and negative affect and the quantity of social interactions, supporting the positivity resonance theory that the individual components alone (i.e., positive affect, social interaction) are necessary but not sufficient for experiencing positivity resonance. Thus, positivity resonance appears to be a distinct and robust motivator of prosocial tendencies. The affective quality of one's social relationships, then, may protect not only the health and well-being of individuals (Major et al., 2018; 
Prinzing et al., in press) but also that of the community members these individuals encounter. In regard to competing predictors of engaging in COVID-19 health behaviors, the association further remained after accounting for demographics, such as age and political orientation, as well as COVID-19related factors such as whether the participant or someone they lived with was considered high-risk of severe illness from COVID-19 and if they had experienced illness symptoms in the last week. This suggests that motivation to engage in health-protective behaviors for the self and community is driven by prosocial, other-oriented concern developed through high-quality social connection, rather than individual threats to health or political ideology.

The strength of the present research is the robustness of findings across different samples and measurement methods, in both cross-sectional and prospective designs. In both studies, we used a latent variable approach to assess prosocial tendencies, which mitigated against the low measurement reliability of any individual indicator. In study 1 , perceived positivity resonance was measured with 2 items in more than 5,000 reported social episodes using the DRM, a less-biased method compared to traditional self-reports (Kahneman \& Krueger, 2006). In study 2, pre-pandemic positivity resonance was measured with 7-items across 7 days, allowing for a marker stable against day-to-day fluctuations in environments and routine, one captured during a time of "normal" social life. Notably, perceived positivity resonance increased slightly $(d=$ .20 ) in the early stage of the pandemic, relative to 2019, indicating that some individuals may have benefited socially (e.g., by spending more quality time with loved ones) during the initial lockdown. Together, our studies demonstrate a robust and generalizable effect of positivity resonance on prosocial tendencies, extending to COVID-19 health behaviors.

Positivity resonance theory holds that accumulated moments of co-experienced positive affect build personal and social resources (i.e., prosocial tendencies). Although the present studies cannot provide causal support for this assertion, via the separation (across assessment periods) of our psychological variables and COVID-19 health behaviors, these studies do establish temporal precedence consistent with this theory-derived causal direction. We suspect, however, that the reverse causal direction is also at play. That is, prosocial tendencies are likely to be a vantage resource in that their presence serves to amplify subsequent experiences of (co-experienced) positive affect, reflecting the reciprocal causality indicative of upward spiral dynamics (Van Cappellen et al., 2018). Another limitation is our exclusive reliance on self-report measures. Although we sought to minimize biases associated with self-reports by using the DRM, this method can be taxing to participants. Additionally, even though our use of a latent measure of prosocial tendencies is a strength, any composite measure is necessarily limited by the set of indicators chosen. One limitation of our selected set of indicators is the lack of standardization in temporal focus (see Table 1). Also, while under "stay-at-home" orders, many days may blend together, potentially making it difficult for participants to recall particular features of the previous day. Future studies might incorporate ecological momentary assessments to capture details of social interactions multiple times within the same day, an approach that may further reduce reporting bias and be less burdensome. While objective measures of positivity resonance such as behavioral (Otero et al., 2019) or physiological (Chen et al., 2020) indicators exist, they are less feasible to collect during a pandemic.

Finally, we did not find strong support for the behavior of social distancing. This may be due to variation across the country in government mandates and degree of viral spread. Alternatively, our measurement approach did not account for people who may have formed "social bubbles"-i.e., repeated contact with the same few people outside the home - which is thought to be a safe and effective way to mitigate spread (Block et al., 2020). Furthermore, while items such as dining out were of great concern early in the pandemic (i.e., April 2020), by T3 (late June 2020) when the final follow-up assessment for study 1 was conducted, many businesses had adapted by expanding outdoor dining, installing plexiglass, or controlling the flow of customers. Thus, some of our social distancing items may not have provided stable reflections of people's effort or consideration of physical distancing. Finally, social distancing is a more complex health behavior (compared to handwashing) in that it may not entirely be in one's control to remain $6 \mathrm{ft}$ away in public settings (e.g., in a store aisle with unpredictable passersby). To the extent that respondents' behavioral intentions did not align with reality, our measure may have misrepresented an individual's motives or concern for others. In sum, our measurement of social distancing in the present study likely posed a limitation.

\section{Conclusion}

Together, two studies offer the first evidence that the affective quality of people's ordinary social interactions in both private and public spaces may shape the enactment of infectionreducing behaviors. These studies contribute to a growing body of work on the importance of prosocial psychological processes for the promotion of public health behaviors such as handwashing and mask wearing during epidemics and pandemics (Pfattheicher et al., 2020; Puterman et al., 2009). Importantly, it expands past work by identifying positivity resonance, a marker of high-quality social connection, as a precursor to prosocial tendencies. Thus, this work is the first to our knowledge to spotlight the role of co-experienced positive affect in promoting adherence to behaviors that promote public health. This evidence bears implications for ongoing and future public health initiatives to address pathogen 
outbreaks in the US and other nations that face similar sociocultural barriers (i.e., "loose" government structures, affective political polarization) and must therefore rely on individuals' prosocial motives to protect that nation's health.

Supplementary Information The online version contains supplementary material available at https://doi.org/10.1007/s42761-021-00035-z.

\section{Additional Information}

Acknowledgements The authors thank Emily Stokes for study 2 data collection, and Kelly Tan and Catherine Berman for their helpful comments on early manuscript drafts.

Funding Information Study 1 was supported by funds from the Department of Psychology and Neuroscience and the College of Arts and Sciences at the University of North Carolina at Chapel Hill, plus a Kenan Distinguished Professorship Fund awarded to Barbara L. Fredrickson. Study 2 was supported by a grant from the Templeton World Charity Foundation (TWCF0325) awarded to Barbara L. Fredrickson.

Data Availability Computed study variables and analytic code are available on the Open Science Framework at https://osf.io/zygq9/?view_only= 5689d9f9c9614d149194eebb1d054b0a.

Conflict of Interests The authors declare no competing interests.

Informed Consent All participants provided informed consent for each survey.

Ethical Approval The Institutional Review Board of the University of North Carolina at Chapel Hill approved all procedures for the study (Study \# 18-2810 and Study \# 20-0841).

\section{References}

Betsch, C., Böhm, R., \& Korn, L. (2013). Inviting free-riders or appealing to prosocial behavior? Game-theoretical reflections on communicating herd immunity in vaccine advocacy. Health Psychology, 32(9), 978-985.

Betsch, C., Böhm, R., Korn, L., \& Holtmann, C. (2017). On the benefits of explaining herd immunity in vaccine advocacy. Nature Human Behaviour, 1(3), 1-6.

Block, P., Hoffman, M., Raabe, I. J., Dowd, J. B., Rahal, C., Kashyap, R., \& Mills, M. C. (2020). Social network-based distancing strategies to flatten the COVID-19 curve in a post-lockdown world. Nature Human Behaviour, 4, 588-596.

Bollen, K. A., \& Stine, R. (1990). Direct and indirect effects: Classical and bootstrap estimates of variability. Sociological Methodology, $20,115-140$.

Boxell, L., Gentzkow, M., \& Shapiro, J. M. (2020). Cross-country trends in affective polarization (No. w26669). Cambridge: National Bureau of Economic Research.

Bremmer, I. (2020). The best global responses to COVID-19 pandemic. Time. Retrieved from https://time.com/5851633/best-globalresponses-covid-19/.

Brown, C. L., Chen, K.-H., Otero, M. C., Wells, J. L., Connelly, D., Levenson, R. W., \& Fredrickson, B. L. (in press). Shared emotions in shared lives: Moments of co-experienced affect, more than individually-experienced affect, linked to relationship satisfaction. Emotion.

Campos, B., Keltner, D., \& Gonzaga, G. C. (2002). Different kinds of love: How love experiences differ across relationships. Irvine: Poster presented at 2002 Western Psychological Association.

Carmines, E. G., \& D'Amico, N. J. (2015). The new look in political ideology research. Annual Review of Political Science, 18, 205-216.

Chen, K. H., Brown, C. L., Wells, J. L., Rothwell, E. S., Otero, M., Levenson, R. W., \& Fredrickson, B. L. (2020). Physiological linkage during shared positive and shared negative emotion. Journal of Personality and Social Psychology.

Cicchetti, D. V. (1994). Guidelines, criteria, and rules of thumb for evaluating normed and standardized assessment instruments in psychology. Psychological Assessment, 6(4), 284-290.

Custers, R., \& Aarts, H. (2005). Positive affect as implicit motivator: On the nonconscious operation of behavioral goals. Journal of Personality and Social Psychology, 89, 129-142.

Diamond, D., \& Wheaton, S. (2020). How the US and Italy traded places on the coronavirus. Politico. Retrieved from https://www.politico. eu/article/how-the-us-and-italy-traded-places-on-coronavirus/

Eisinga, R., Te Grotenhuis, M., \& Pelzer, B. (2013). The reliability of a two-item scale: Pearson, Cronbach, or Spearman-Brown? International Journal of Public Health, 58(4), 637-642.

Elliot, A. J., \& Sheldon, K. M. (1998). Avoidance personal goals and the personality-illness relationship. Journal of Personality and Social Psychology, 75(5), 1282-1299.

Fincher, C. L., Thornhill, R., Murray, D. R., \& Schaller, M. (2008). Pathogen prevalence predicts human cross-cultural variability in individualism/collectivism. Proceedings of the Royal Society B: Biological Sciences, 275(1640), 1279-1285.

Fredrickson, B. L. (2013). Positive emotions broaden and build. In Advances in experimental social psychology (Vol. 47, pp. 1-53). Cambridge: Academic Press.

Fredrickson, B. L. (2016). Love: Positivity resonance as a fresh, evidence-based perspective on an age-old topic. In L. F. Barrett, M. Lewis, \& J. M. Haviland-Jones (Eds.), Handbook of emotions (4th ed., pp. 847-858). New York: Guilford Press.

Fredrickson, B. L., \& Joiner, T. (2018). Reflections on positive emotions and upward spirals. Perspectives on Psychological Science, 13(2), 194-199.

Gelfand, M. J., Raver, J. L., Nishii, L., Leslie, L. M., Lun, J., Lim, B. C., et al. (2011). Differences between tight and loose cultures: A 33nation study. Science, 332(6033), 1100-1104.

Harrell Jr., F. E., Lee, K. L., \& Mark, D. B. (1996). Multivariable prognostic models: Issues in developing models, evaluating assumptions and adequacy, and measuring and reducing errors. Statistics in medicine, 15(4), 361-387.

Igielnik, R. (2020). Most Americans say they regularly wore a mask in stores in the past month, fewer see others doing it. Pew Research Center. Retrieved from https://www.pewresearch.org/fact-tank/ 2020/06/23/most-americans-say-they-regularly-wore-a-mask-instores-in-the-past-month-fewer-see-others-doing-it/

Ingoglia, S., Lo Coco, A., \& Albiero, P. (2016). Development of a brief form of the Interpersonal Reactivity Index (B-IRI). Journal of Personality Assessment, 98(5), 461-471.

Kahneman, D., \& Krueger, A. B. (2006). Developments in the measurement of subjective well-being. Journal of Economic Perspectives, 20(1), 3-24.

Kahneman, D., Krueger, A. B., Schkade, D. A., Schwarz, N., \& Stone, A. A. (2004). A survey method for characterizing daily life experience: The day reconstruction method. Science, 306(5702), 1776-1780.

Kline, R. B. (2015). Principles and practice of structural equation modeling. Cambridge: Guilford Publications.

Lapsley, D. K., \& Narvaez, D. (2014). The having, doing and being of moral personality. In The philosophy and psychology of character and happiness (pp. 133-159). 
Lee-Baggley, D., DeLongis, A., Voorhoeave, P., \& Greenglass, E. (2004). Coping with the threat of severe acute respiratory syndrome: Role of threat appraisals and coping responses in health behaviors. Asian Journal of Social Psychology, 7(1), 9-23.

Levy, J., Goldstein, A., \& Feldman, R. (2019). The neural development of empathy is sensitive to caregiving and early trauma. Nature Communications, 10(1), 1-10.

Major, B. C., Le Nguyen, K. D., Lundberg, K. B., \& Fredrickson, B. L. (2018). Well-being correlates of perceived positivity resonance: Evidence from trait and episode-level assessments. Personality and Social Psychology Bulletin, 44(12), 1631-1647.

Moncus, J. J., \& Connaughton, A. (2020). Americans' views on World Health Organization split along partisan lines as Trump calls for U.S. to withdraw. Pew Research Center. Retrieved from https:// www.pewresearch.org/fact-tank/2020/06/11/americans-views-onworld-health-organization-split-along-partisan-lines-as-trump-callsfor-u-s-to-withdraw/

Nai, J., Narayanan, J., Hernandez, I., \& Savani, K. (2018). People in more racially diverse neighborhoods are more prosocial. Journal of Personality and Social Psychology, 114(4), 497-515.

Nevitt, J., \& Hancock, G. R. (2001). Performance of bootstrapping approaches to model test statistics and parameter standard error estimation in structural equation modeling. Structural Equation Modeling, 8(3), 353-377.

Otero, M. C., Wells, J. L., Chen, K. H., Brown, C. L., Connelly, D. E., Levenson, R. W., \& Fredrickson, B. L. (2019). Behavioral indices of positivity resonance associated with long-term marital satisfaction. Emotion, 20, 1225-1233. https://doi.org/10.1037/emo0000634.

Pargament, K. I., Oman, D., Pomerleau, J., \& Mahoney, A. (2017). Some contributions of a psychological approach to the study of the sacred. Religion, 47(4), 718-744.

Pfattheicher, S., Nockur, L., Böhm, R., Sassenrath, C., \& Petersen, M. B. (2020). The emotional path to action: Empathy promotes physical distancing and wearing of face masks during the COVID-19 pandemic. Psychological Science, 31, 1363-1373.

Piedmont, R. L. (1999). Does spirituality represent the sixth factor of personality? Spiritual transcendence and the five-factor model. Journal of Personality, 67(6), 985-1013.

Preston, S. D., \& De Waal, F. B. (2002). Empathy: Its ultimate and proximate bases. Behavioral and Brain Sciences, 25(1), 1-20.

Prinzing, M. M., Zhou, J., West, T. N., Le Nguyen, K. D., \& Fredrickson, B. L. (in press). Staying 'in sync' with others during COVID-19: Positivity resonance mediates cross-sectional and longitudinal links between trait resilience and mental health. The Journal of Positive Psychology.

Puterman, E., Delongis, A., Lee-Baggley, D., \& Greenglass, E. (2009). Coping and health behaviours in times of global health crises: Lessons from SARS and West Nile. Global Public Health, 4(1), 69-81.

Robinson, M. D., \& Clore, G. L. (2002). Belief and feeling: evidence for an accessibility model of emotional self-report. Psychological Bulletin, 128(6), 934-960.
Rosseel, Y. (2012). lavaan: An R package for structural equation modeling. Journal of Statistical Software, 48(2), 1-36 http://www. jstatsoft.org/v48/i02/.

Rushton, J. P., Chrisjohn, R. D., \& Fekken, G. C. (1981). The altruistic personality and the self-report altruism scale. Personality and Individual Differences, 2(4), 293-302.

Saroglou, V., Buxant, C., \& Tilquin, J. (2008). Positive emotions as leading to religion and spirituality. The Journal of Positive Psychology, 3(3), 165-173.

Sassenrath, C., Diefenbacher, S., Siegel, A., \& Keller, J. (2016). A person-oriented approach to hand hygiene behaviour: Emotional empathy fosters hand hygiene practice. Psychology \& Health, 31(2), 205-227.

Schaller, M., \& Park, J. H. (2011). The behavioral immune system (and why it matters). Current Directions in Psychological Science, 20(2), 99-103.

Schreiber, J. B., Nora, A., Stage, F. K., Barlow, E. A., \& King, J. (2006). Reporting structural equation modeling and confirmatory factor analysis results: A review. The Journal of Educational Research, 99(6), 323-338.

Shim, E., Chapman, G. B., Townsend, J. P., \& Galvani, A. P. (2012). The influence of altruism on influenza vaccination decisions. Journal of the Royal Society Interface, 9(74), 2234-2243.

Shiota, Michelle (2020). RAPID: Coping with COVID-19: Emotion regulation strategy implications for behavior, social outcomes, and well-being. National Science Foundation, Award Abstract \#202861. Retrieved from: https://www.nsf.gov/awardsearch/ showAward?AWD_ID=2028261\&HistoricalAwards=false

Sprecher, S., \& Fehr, B. (2005). Compassionate love for close others and humanity. Journal of Social and Personal Relationships, 22(5), 629-651.

Underwood, L. G., \& Teresi, J. A. (2002). The daily spiritual experience scale: Development, theoretical description, reliability, exploratory factor analysis, and preliminary construct validity using healthrelated data. Annals of Behavioral Medicine, 24(1), 22-33.

Valdesolo, P., \& DeSteno, D. (2011). Synchrony and the social tuning of compassion. Emotion, 11(2), 262-266.

Van Bavel, J. J., Baicker, K., Boggio, P. S., Capraro, V., Cichocka, A., Cikara, M., et al. (2020). Using social and behavioural science to support COVID-19 pandemic response. Nature Human Behaviour, 4, 460-471.

Van Cappellen, P., Rice, E. L., Catalino, L. I., \& Fredrickson, B. L. (2018). Positive affective processes underlie positive health behaviour change. Psychology \& Health, 33(1), 77-97.

Van Cappellen, P., Saroglou, V., Iweins, C., Piovesana, M., \& Fredrickson, B. L. (2013). Self-transcendent positive emotions increase spirituality through basic world assumptions. Cognition \& Emotion, 27(8), 1378-1394.

Zhou J., Prinzing, M.M., Le Nguyen, K.D., West, T.N., \& Fredrickson, B.L. (revision invited). The goods in everyday love: Do increases in positivity resonance increase communal virtues? 